\title{
An introduction to the bootstrap: a versatile method to make inferences by using data-driven simulations
}

\author{
Guillaume A. Rousselet ${ }^{1}$, Cyril R. Pernet ${ }^{2}$, Rand R. Wilcox ${ }^{3}$ \\ 1: Guillaume.Rousselet@glasgow.ac.uk School of Psychology and Neuroscience, University of Glasgow, Glasgow, \\ Scotland, G12 8QB, UK \\ 2: wamcyril@gmail.com Neurobiology Research Unit, Copenhagen University Hospital, Rigshospitalet, DK-2100 \\ Copenhagen, Denmark \\ 3: rwilcox@usc.edu Department of Psychology, University of Southern California, Los Angeles, CA, USA
}

\begin{abstract}
The bootstrap is a versatile technique that relies on data-driven simulations to make statistical inferences. When combined with robust estimators, the bootstrap can afford much more powerful and flexible inferences than is possible with standard approaches such as t-tests on means. In this tutorial, we use detailed illustrations of bootstrap simulations to give readers an intuition of what the bootstrap does and how it can be applied to solve many practical problems, such as building confidence intervals for many aspects of the data. In particular, we illustrate how to build confidence intervals for measures of location, including measures of central tendency, in the one-sample case, for two independent and two dependent groups. We also demonstrate how to compare correlation coefficients using the bootstrap and to perform simulations to determine if the bootstrap is fit for purpose for a particular application. Our approach is to suggest and motivate what could be done in a situation, with an understanding that various options are valid, though they may help answer different questions about a dataset. The tutorial also addresses two widespread misconceptions about the bootstrap: that it makes no assumptions about the data, and that it leads to robust inferences on its own. The tutorial focuses on detailed graphical descriptions, with data and code available online to reproduce the figures and analyses in the article (OSF: https://osf.io/8b4t5/; GitHub: https://github.com/GRousselet/bootstrap).
\end{abstract}

Accepted in Meta-Psychology.

The fully transparent Editorial process can be accessed by clicking here. Anyone can contribute with Open Peer Review. To do so, directly add comments into the PDF via hypothes.is." 


\section{Introduction}

The bootstrap is a well-established early computer-age inferential method (Efron, 1979; Efron \& Hastie, 2016; Efron \& Tibshirani, 1994). The bootstrap is based on the idea that using only the data at hand can sometimes give better results than making unwarranted assumptions about the populations we're trying to estimate. The core mechanism of the bootstrap is sampling with replacement from the data, which is a form of data-driven simulation. Thus, learning about the bootstrap is not only learning about an alternative to the standard parametric methods of statistical inference, but is also a way to learn about simulations and to question our choices of methods. As we will see, the bootstrap doesn't provide a single alternative approach to classic problems such as group comparisons, but a large family of new approaches. Having the bootstrap in your toolbox is like getting a powerful Swiss Army Knife, which offers great versatility - others have referred to the bootstrap as Meat Axe, Swan-Dive, Jack-Rabbit, and Shotgun (Efron, 1979).

In this tutorial, we start by explaining the basic mechanism of the bootstrap and the rich output it creates. We then consider the limitations of the bootstrap, before covering key applications to inferences about group comparisons of measures of locations and correlation coefficients. We also provide examples of simulations to quantify the long-term behaviour of the bootstrap, to help make informed choices about statistical tools. Finally, we briefly introduce one of many variants of the original bootstrap, the bootstrap-t technique, which has applications in analyses of variance (ANOVAs). Other variants of the bootstrap have been proposed since 1979, such as the wild bootstrap ( $\mathrm{Wu}, 1986)$, the bias-corrected and accelerated bootstrap, the smooth bootstrap (Efron \& Tibshirani, 1994), and the Bayesian bootstrap (Bååth, 2015; Rubin, 1981), to name a few - and research into bootstrap methods is still very active.

Before we start, let's keep in mind that for each combination of experimental design and type of data, there is a variety of statistical analysis options available, each asking different questions, and that no method dominates in all situations (see e.g. Rousselet et al., 2017; Rousselet \& Wilcox, 2019). The bootstrap is no exception: it works well in some situations but not in others. 


\section{How to read this article}

This tutorial is pitched at a rather conceptual level and covers a lot of statistical methods and concepts. Readers interested in a gentler introduction, including a step-by-step guide of the bootstrap implementation in $R$ (R Core Team, 2021), should consider our earlier tutorial on the topic with its associated reproducibility package (Rousselet et al., 2019, 2021). After reading this article, or if you already know the basics, come back here for a more in-depth coverage of the topic. While the current article can be read on its own, interested readers can also access, in separate notebooks, all the code matching the simulations and figures in the article. To facilitate this process, each figure caption contains a link to an html version of the matching notebook on GitHub (https: / /github.com/GRousselet/bootstrap), and a pdf version of the notebook and of the figure on the OSF (https://osf.io/8b4t5/). The notebooks are also available as .Rmd files on the OSF. Both repositories contain a README file that lists what to expect from the 9 notebooks, the main $\mathrm{R}$ packages needed and various resources.

\section{Disclosures}

All the figures are licensed CC-BY 4.0 and are available as separate pdf files. Each figure caption ends with links to its matching pdf and the RMarkdown notebook that can be used to reproduce it. The main $R$ packages used to generate the data and make the figures are RGenData (Ruscio \& Kaczetow, 2008), LambertW(Goerg, 2011, 2022), MASS (Venables \& Ripley, 2002), gsl (Hankin, 2006), nleqslv (Hasselman, 2022), ggplot2 (Wickham, 2016), cowplot (Wilke, 2017), tibble (Müller \& Wickham, 2018), cubelyr (Wickham, 2020), facetscales (Moreno, 2019), rogme (Rousselet et al., 2017), knitr (Xie, 2018), and the essential beepr (Bååth, 2018).

\section{Bootstrap: the basics}

Imagine we carried out an experiment and we obtained the 30 observations in Figure 1A. The traditional approach to compute a confidence interval (CI in the rest of the text) involves a few 
calculations and some parametric assumptions. First, some null value is subtracted from the mean (let say here our null hypothesis is that the population mean is 2), and this difference is then normalised by dividing it by the standard error of the mean (SEM). This $\mathcal{T}$ statistic (mean difference/SEM) is assumed to have a particular long-run distribution determined by the sample size (i.e. if we were to redo this experiment many times, with 30 observations, this is the distribution we should get). This distribution, also known as a $T$ distribution, here with 29 degrees of freedom, is illustrated in Figure 1B. The distribution assumes that we repetitively sample from a standard normal distribution, and for each sample of size $n=30$, we compute a $T$ value. To build a 95\% CI, we read out the 2.5th quantile from that distribution and plug that value into the CI formula (Figure 1B inset). In our example, the sample mean is 1.61 and a 95\% CI, which contains the null value, is $[0.9,2.31]$. A CI for the mean is an interval that covers the population mean with a rate of error alpha, here 5\% for a 95\% CI (Morey \& Rouder, 2011). That is, across many repetitions of the same experiment, $95 \%$ of such intervals will contain the population value we are trying to estimate. There is no guarantee that the interval obtained in one experiment contains the population value, and there is no probability or confidence associated with a single interval: it contains the population value or not. The $95 \%$ only applies to an infinitely long collection of intervals. A CI can also be described as the interval compatible with the data, given our model (i.e. the long-run $\mathcal{T}$ distribution and any other experimental and statistical assumptions we make) - so when the model is wrong, the interval will not behave as intended in the long-run (Greenland et al., 2016). We will demonstrate how to assess the validity and quality of CIs using simulations in later sections.

Finally, the probability of observing a result at least as extreme as our $T$ value, given the null long-run $T$ distribution, is the $P$ value (Pernet, 2017). Half of the $P$ value is the area under the curve marked in red in Figure 1B, to the left of the observed $T$ value. To obtain the $P$ value for a two-sided test, the area is multiplied by two. Note that there is a direct relationship between CIs and $P$ values: a 95\% CI contains all the hypotheses / population values for which the $P$ value is larger than 0.05. Hypotheses outside a 95\% CI are associated with $P$ values inferior to 0.05 . 
A

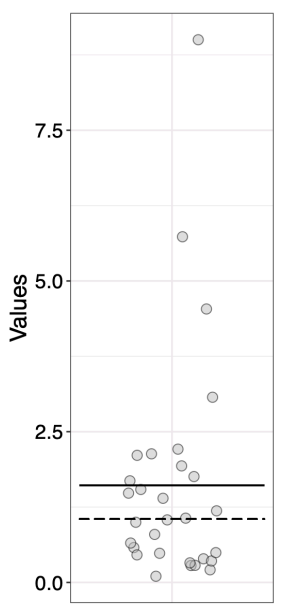

B

$T$ distribution with 29 degrees of freedom

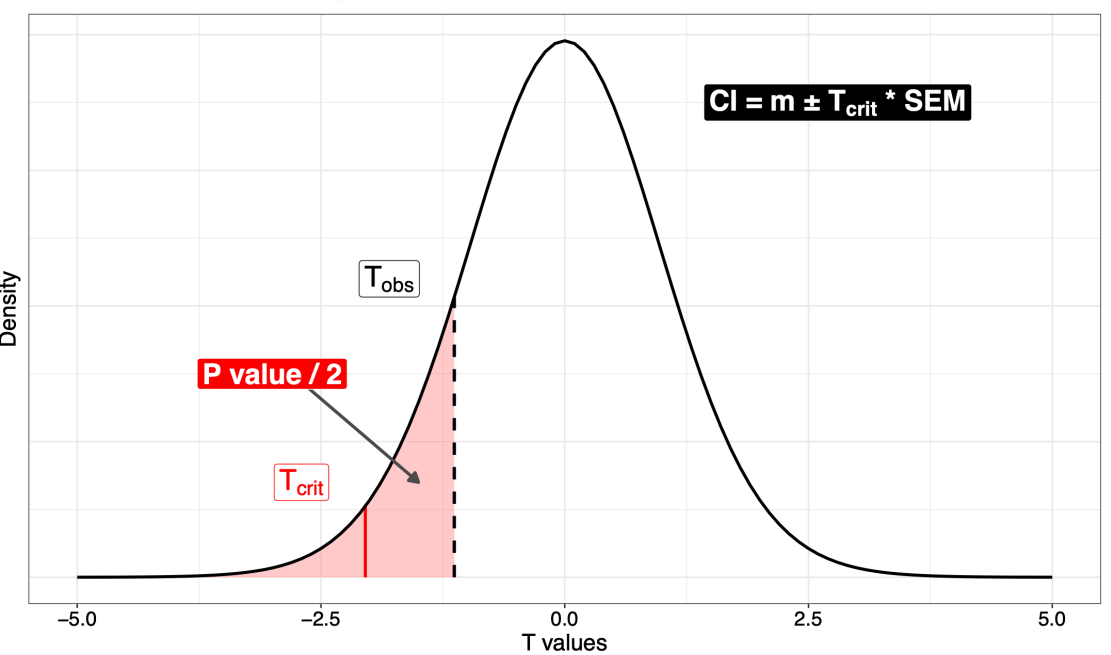

C

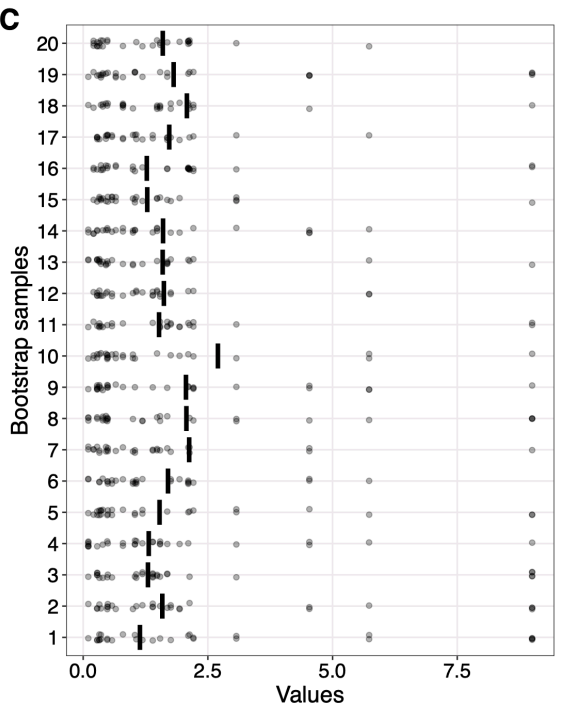

D

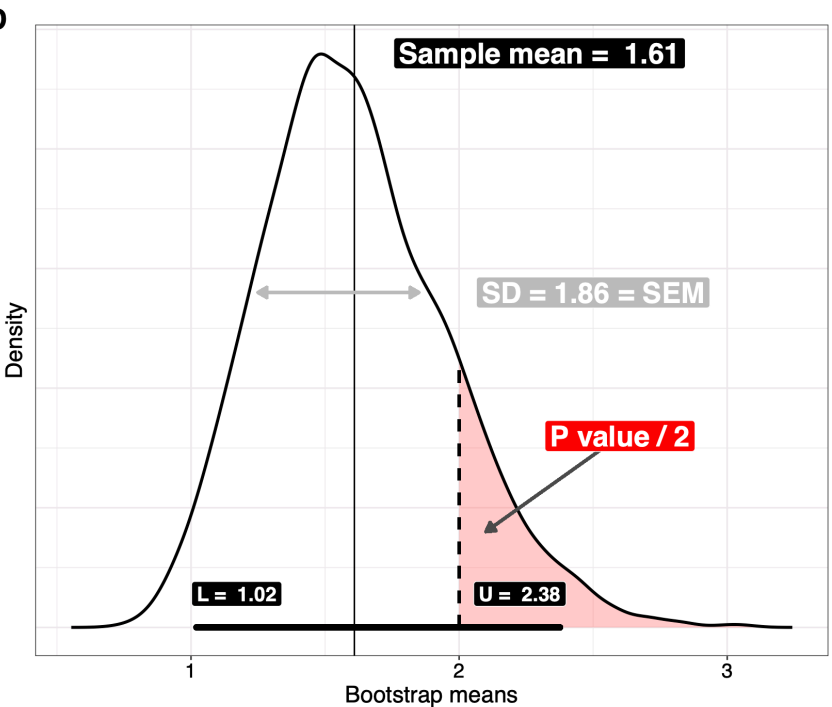

Figure 1. Percentile bootstrap inferences. A. Stripchart illustrating a random sample $(\mathrm{n}=30)$ from a skewed (lognormal) distribution. Each disk is an observation. The horizontal dashed line marks the sample median; the plain line marks the sample mean. B. Sampling $T$ distribution for 29 degrees of freedom. Tcrit $=$ critical $T$ value; Tobs $=$ observed $T$ value. The red area corresponds to half the $P$ value. The inset contains the formula for the standard $T$-test confidence interval. G. Bootstrap samples. Each disk is a bootstrap observation. For each bootstrap sample, the vertical line marks the bootstrap mean. D. Bootstrap sampling distribution of the mean. The standard deviation (SD) of the bootstrap distribution provides an estimate of the standard error of the mean (SEM). The $P$ value is equal to twice the minimum between the proportion of bootstrap samples to the left and to the right of the null value (here 2), marked by the vertical dashed line. So in this example the $P$ value is twice the red area for a two-sided test. The confidence interval is marked by a black horizontal line, with the values of the lower $(\mathrm{L})$ and upper $(\mathrm{U})$ bounds in black labels. This figure is available as a separate pdf file. It was created using the $\mathrm{R}$ notebook $p b$, which is available in pdf,$\underline{\mathbf{h t m l}}$ and $\underline{\text { Rmd }}$ formats. 
An interesting aspect from the example above is that when we use a $T$ - test (or another parametric test), it assumes a certain long-term distribution of the test statistics. Indeed, the confidence interval based on the $T$-test relies on the long-term value of $\mathcal{T}$. Suppose, for example, that a study were repeated many times and that $2.5 \%$ of these $T$ values are less than or equal to 2 and $2.5 \%$ are greater than or equal to 2 . Then the .95 CI would be given by the formula in Figure 1B insert, with Tcrit=2. Assuming normality, there is no need to determine the critical value in this manner; the critical value can be derived exactly. But momentarily imagine that the critical value is unknown. One could determine it by generating data from a normal distribution, note the value of $T$, and repeat this process say 10,000 times. If $2.5 \%$ of these $T$ values are less than or equal to -2 , and $2.5 \%$ are greater than or equal to 2 , this indicates that Tcrit $=2$. This result is based on a particular model: random sampling from a normal distribution. Thus, longterm $T$ distributions used in $T$-tests (and $F$ distributions in ANOVAs) represent the outcome of virtual experiments, given a certain sample size and assuming that we sample from a population with a particular shape. As we will see, the bootstrap-t method, described later in this article, mimics this process. The main difference is that values are randomly sampled with replacement from the observed data rather than from a normal distribution.

With the bootstrap, we relax parametric assumptions to instead perform data-driven simulations. The core mechanism of the bootstrap is random sampling with replacement. To illustrate, say we have this sample of 6 observations: 1, 2, 3, 4, 5, 6. A bootstrap sample consists of 6 observations sampled with replacement from the original ones. Here are examples of three such bootstrap samples:

[1] 123633

[2] 354664

[3] 132625

In some bootstrap samples, some original observations are sampled more than once, others are not sampled at all. For each bootstrap sample, we compute an estimate, say the sample mean. So, if we take 1,000 bootstrap samples and for each of them we compute the mean, we end up with a distribution of 1,000 bootstrap estimates of the sample mean. The key idea is that if the 
original sample size and the number of bootstrap samples are large enough, in many situations the distribution of bootstrap estimates provides a good approximation of the sampling distribution of the estimate.

If we go back to our Figure 1 example, panel $\mathrm{C}$ illustrates 20 bootstrap samples. Given our sample size of $n=30$, each of these bootstrap samples contains 30 observations sampled with replacement from the original sample. Because of random sampling, the bootstrap mean differs across bootstrap samples. The idea is that these fluctuations represent the fluctuations expected if we repeated the same experiment many times, each time collecting 30 new observations and computing the mean. After 5,000 bootstraps, we obtain the distribution in Figure 1D. This sampling distribution of bootstrap estimates is the main outcome of the bootstrap procedure. From this distribution, we can derive five important elements:

- $\quad$ an estimate of the shape of the sampling distribution;

- $\quad$ an estimate of the standard error of the quantity (here the mean);

- $\quad$ an estimate of bias;

- a confidence interval;

- a $P$ value.

All of that without equations or parametric assumptions, and for any quantity! Indeed, if the standard $T$ - test is restricted to inferences on means, the bootstrap can be used to build a CI about any estimate (e.g. mean, median, or any quantile), making it very practical in situations where there is no analytical solution. Because the bootstrap distribution contains so much information, and much more than the CI derived from it, when possible we recommend illustrating the full distribution in articles.

Here the bootstrap CI is $[1.02,2.38]$, which is very similar to the one we got using the standard T-test formula- $[0.9,2.31]$. It is obtained by getting the quantiles of the bootstrap distribution. For instance, for a 95\% CI, the lower bound is defined as the 2.5th quantile of the bootstrap distribution, and the upper bound at the 97.5th quantile. Because the bootstrap distribution is an 
estimate of the sampling distribution, its standard deviation provides an estimate of the standard error, here of the mean. The bootstrap distribution is positively skewed (skewed to the right), correctly suggesting that the sampling distribution of the mean is asymmetric. This is correct because we draw the data from a lognormal distribution and not from a normal distribution, as assumed by the $T$ - distribution in Figure $1 \mathrm{~B}$.

The $P$ value is computed as the minimum of the proportion of bootstrap means larger than the null (2) and the proportion of bootstrap means smaller than the null, multiplied by two (here bootstrap $P=0.276)$. Intuitively, it reflects how deeply the null value is nested within the bootstrap distribution. When the null value is exactly in the middle of the bootstrap distribution, the $P$ value is 1 , when it falls completely outside the distribution, the $P$ value is 0 . Finally, readers interested in bias and its bootstrap estimation will find detailed illustrations and code in another article (Rousselet \& Wilcox, 2019).

The bootstrap method described above is called the percentile bootstrap, first described in 1979 in Bradley Efron's seminal paper (Efron, 1979). Since 1979, many other bootstrap techniques have been developed, and we will describe only one other version in a later section: the bootstrap-t technique (also referred to as percentile-t bootstrap), in which the bootstrap is used to compute a data-driven $T$ distribution under the null hypothesis. More in depth coverage of the bootstrap and its many variants is available in several books and articles (Efron \& Tibshirani, 1986, 1994; Hesterberg, 2015; Wilcox, 2017; Wilcox \& Keselman, 2003). In the rest of this tutorial, we refer to the percentile bootstrap as the bootstrap for short.

As illustrated already, the bootstrap is a data-driven (we only use the data at hand) and nonparametric (we do not assume the data can be modelled by a distribution with a fix set of parameters) method, and if the original sample size and the number of bootstrap samples are large enough, the bootstrap distribution does a good job at capturing the shape of the sampling distribution of the quantity of interest. The data-driven, non-parametric aspect means that the bootstrap cannot suggest impossible confidence bounds, unlike standard parametric methods. For instance, consider the percentage correct data in Figure 2. Panel A illustrates data and standard confidence intervals. Condition 1 shows a sample well spread out with a mean around 74. In condition 2, most participants are near the ceiling. In condition 3, the lowest value from 
condition 2 was replaced by an outlier with a score of 60 . Now the upper bound of the confidence interval is slightly over $100 \%$, which is of course impossible. This is because the model used to build the confidence interval is incorrect in this case: it assumes sampling from continuous and symmetric distributions, whereas our sample comes from a bounded and asymmetric distribution. Unfortunately, in our experience, impossible CIs are common in publications and presentations. In contrast, the bootstrap makes no parametric assumptions about the population our samples come from and instead uses the sample only, such that the bootstrap confidence intervals cannot, by definition, extend beyond the smallest or largest observations in our sample (Figure 2B).
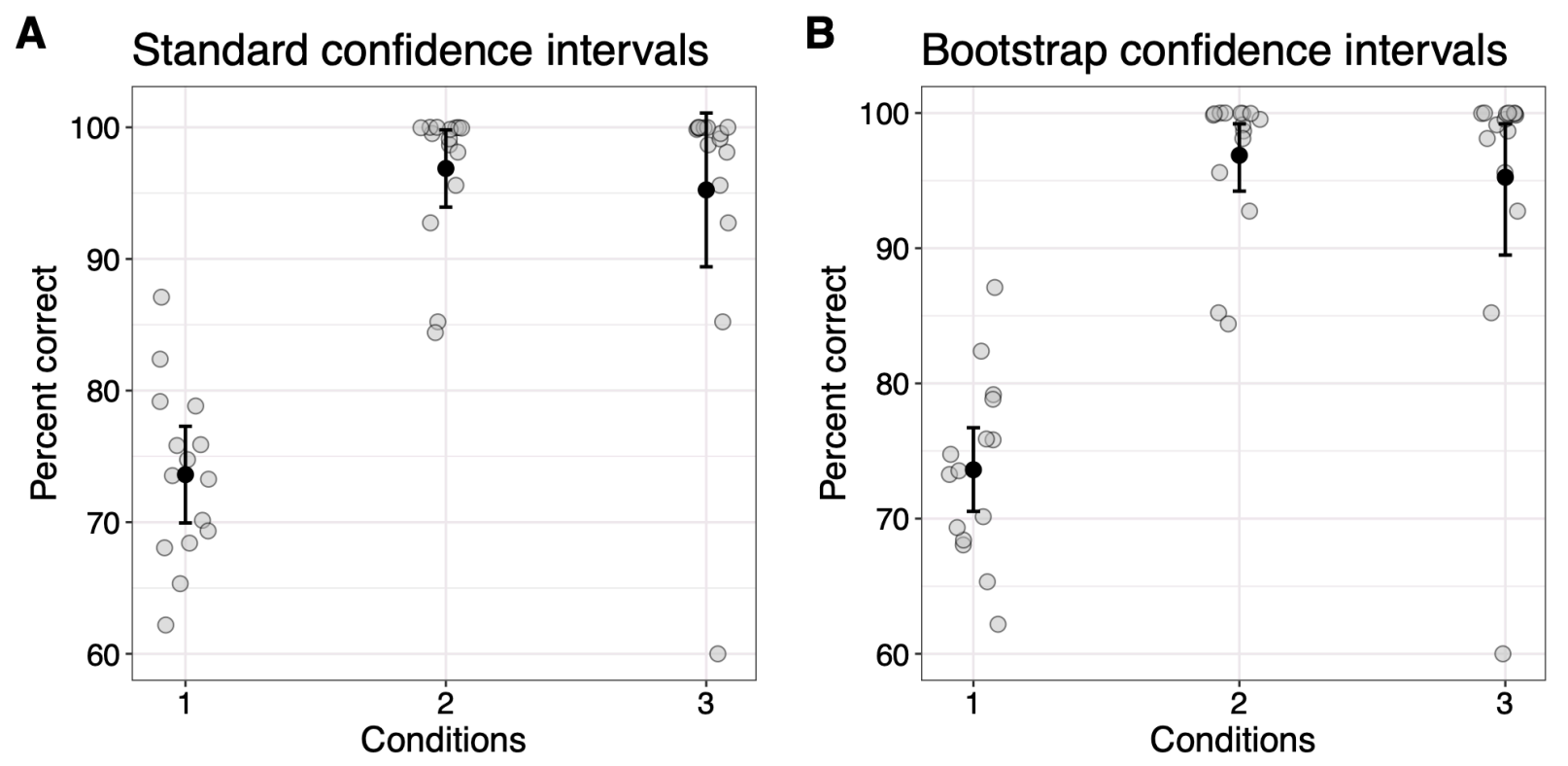

Figure 2. Application of the bootstrap to percent correct data. Each grey disk is an observation. The black disk marks the sample mean. The error bars mark the $95 \%$ confidence intervals, computed using the standard $\mathcal{T}$ - test equation $(\mathbf{A})$ or the bootstrap $(\mathbf{B})$. This figure is available as a separate pdf file. It was created using the $\mathrm{R}$ notebook $p c$, which is available in pdf $\underline{\mathbf{h t m l}}$ and $\underline{\mathbf{R m \boldsymbol { d }}}$ formats.

To illustrate that the bootstrap can provide a good approximation of sampling distributions, let's consider a population with a standard lognormal distribution (Figure 3). This distribution is positively skewed, and it is has a zero lower bound (only positive values can be observed), as one could observe for instance with reaction times, fixation durations, pupil diameter, BMI etc. As 
such, it provides a much better example of the sort of continuous distributions we encounter in psychology and in neuroscience. More generally in the social and life sciences, many quantities have a skewed distribution similar to the one shown in Figure 3 (Limpert et al., 2001; Limpert \& Stahel, 2017).

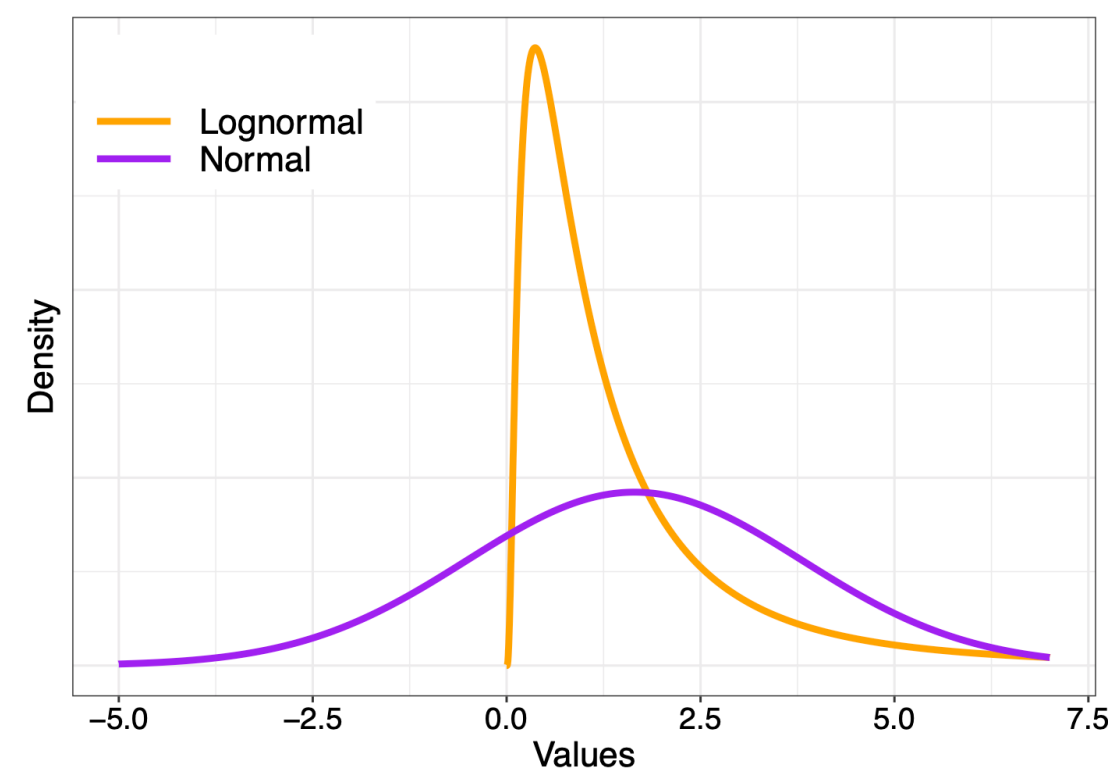

Figure 3. Lognormal and normal distributions. The two distributions have the same mean and the same variance. Unlike the normal distribution, the lognormal distribution is skewed and bounded, similarly to most quantities we measure in psychology and in neuroscience. This figure is available as a separate pdf file. It was created using the $\mathrm{R}$ notebook sampdist, which is available in pdf $\underline{\mathbf{h t m l}}$ and $\underline{\text { Rmd }}$ formats.

To visualise examples of sampling distributions, we take 50,000 random samples from a lognormal distribution and each time compute some statistics (Figure 4A). We could compute the mean, but it is only one of many options to quantify the location of a distribution, and not a good one when there is skewness because it can reflect a highly atypical response (Rousselet \& Wilcox, 2019). More generally, one has to go beyond the mean to understand how distributions differ (Rousselet et al., 2017). So here, as an example, we compute the $20 \%$ trimmed mean, which provides a robust measure of location (Wilcox, 2017). 
To compute a 20\% trimmed mean, observations are sorted, the lower and upper $20 \%$ are discarded and the remaining observations are averaged. In this context, the mean is a $0 \%$ trimmed mean and the median is a 50\% trimmed mean. In our example, we compute 50,000 trimmed means for sample sizes $n=20,30$ and 50. In other words, we look at how the $20 \%$ trimmed mean is distributed when we perform many experiments with certain sample sizes. These sampling distributions are usually unobservable, but we can visualise them here because we performed simulations. As shown in the top row of Figure 4 (panel A), all sampling distributions are positively skewed, even for $n=50$. They also get narrower with increasing sample size, because the larger the sample, the closer on average each experimental estimate is to the population value.

The bootstrap aims to estimate the shape of these distributions. In each column of Figure 4, under the sampling distribution, four of the 50,000 samples are illustrated (panel B). For each sample, 5,000 bootstrap estimates are computed. In all cases, the bootstrap distributions suggest, correctly, that the sampling distribution is positively skewed. The exact shape of each bootstrap sampling distribution is dictated by the sample at hand, and no other information is considered. Because of this, some bootstrap distributions are more skewed if the samples contain more extreme values - for instance contrast samples 1 and 4 in column 1. With increasing sample sizes, the bootstrap distributions also tend to be narrower, mirroring the behaviour of the sampling distributions. 

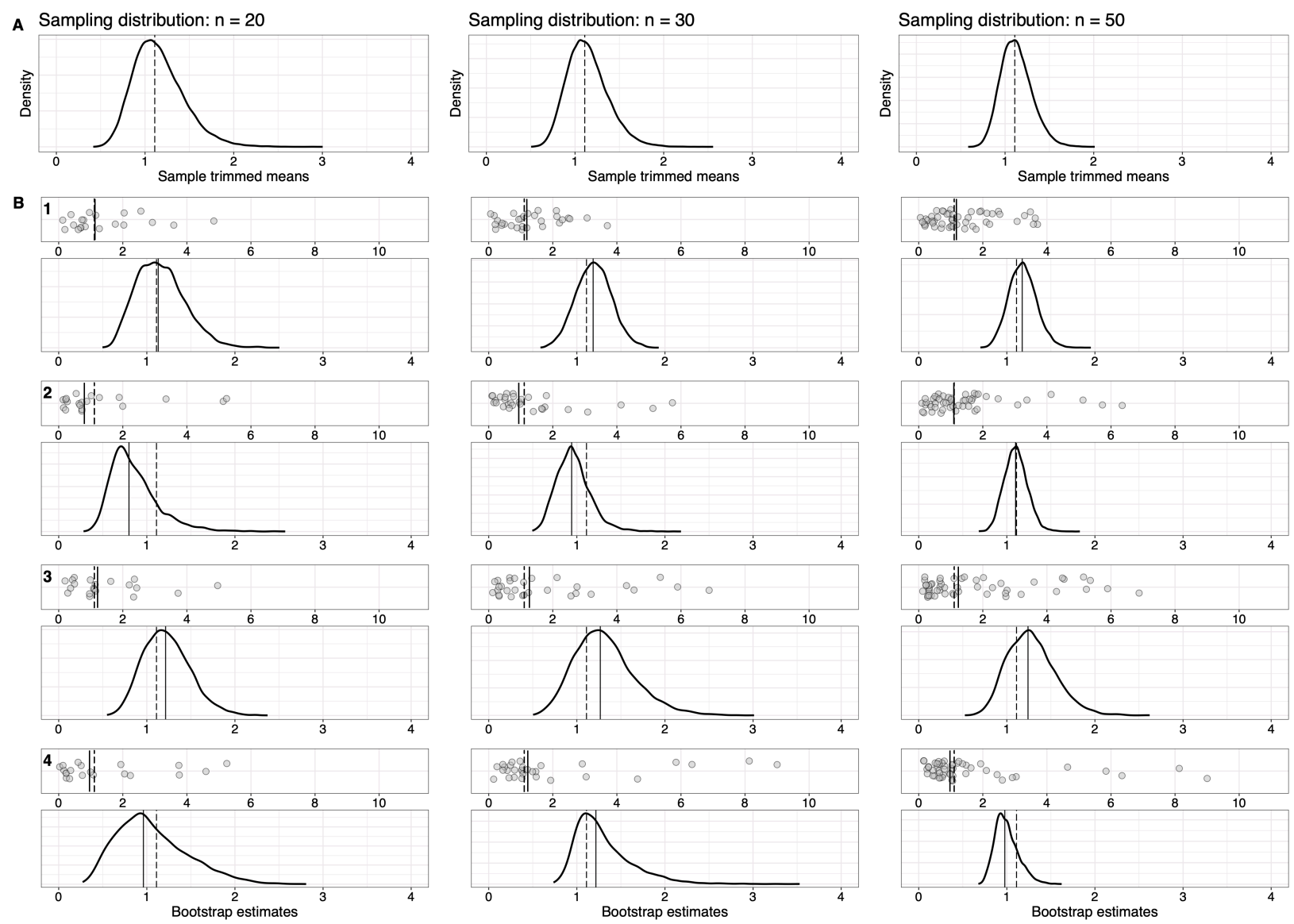

Figure 4. Bootstrap estimates of sampling distributions. (A) The top row shows sampling distributions of the $20 \%$ trimmed mean for sample size of $n=20, n=30$ and $n=50$. These distributions were obtained by taking 50,000 samples from the lognormal distribution illustrated in Figure 3 . The vertical dashed lines mark the population $20 \%$ trimmed mean. (B) In each column, the dyads of rows under the sampling distribution show 4 of the 50,000 samples. In each dyad, the narrow panel contains a scatterplot of the observations in the sample. The vertical black line marks the sample $20 \%$ trimmed mean. The lower panel shows the bootstrap distribution of the $20 \%$ trimmed mean, based on 5,000 bootstrap samples. This figure is available as a separate pdf file and was inspired by the illustrations in (Hesterberg, 2015). It was created using the $\mathrm{R}$ notebook sampdist, which is available in pdf $\underline{\mathbf{h t m l}}$ and .Rmd formats.

Figure 4 also provides an important reminder about bootstrap inferences. Each bootstrap distribution is centred around the sample estimate, not the population value, so the bootstrap can improve inferences, not estimation (Hesterberg, 2015). Moreover, bootstrap CIs, like any other CIs, vary across experiments (Figure 5). Therefore, if we perform a single experiment, the CI we 
obtain does or does not contain the population value we're trying to estimate. The coverage probability (say 95\%) is only defined in the long run; there is no guarantee for a single experiment (Greenland et al., 2016). And as we will see later, the actual coverage can be quite different from the intended one- for instance an intended 95\% CI can, in some situations, be a 90\% CI.

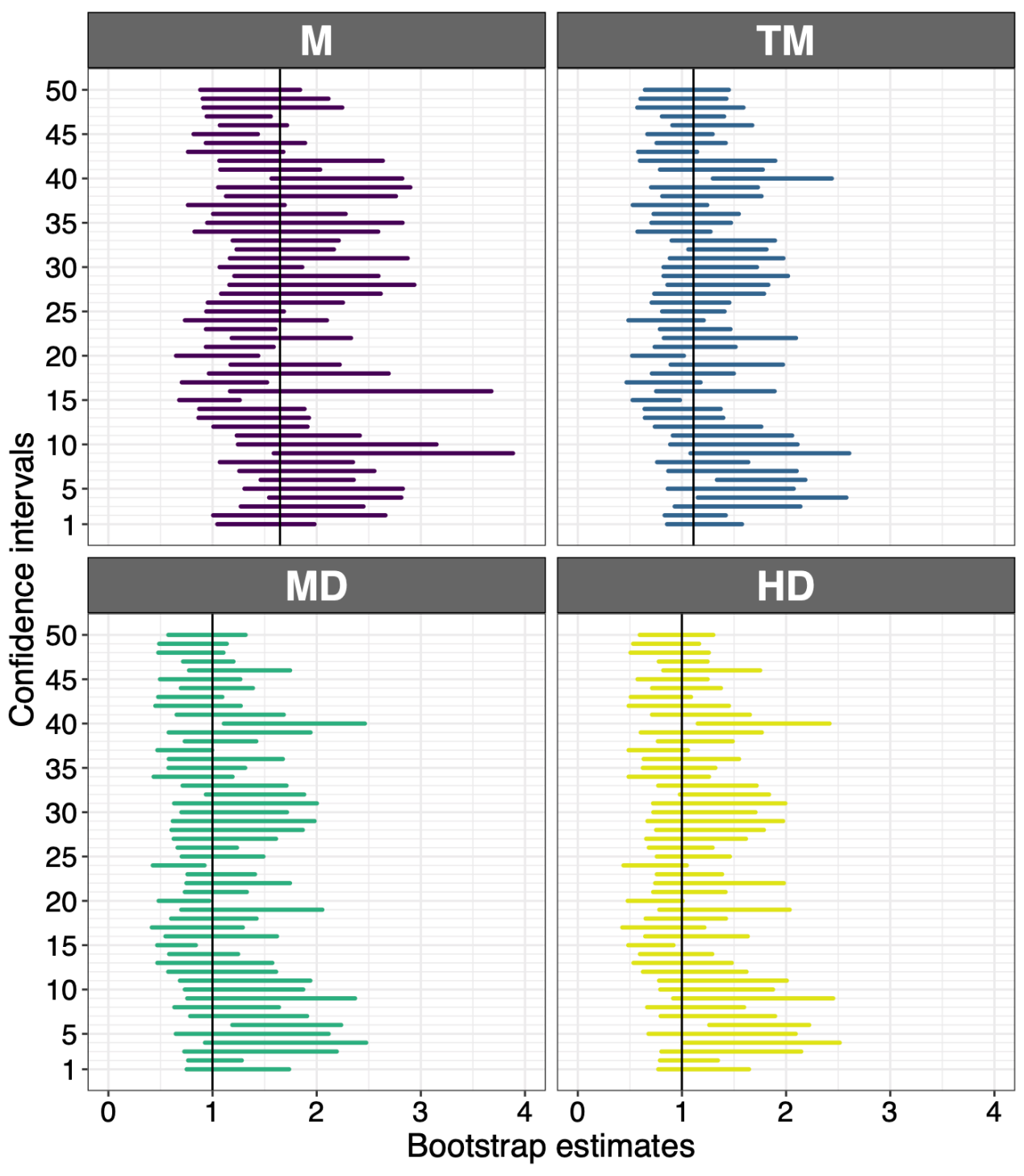

Figure 5. 50 experiments, 50 bootstrap confidence intervals. For each experiment, a random sample of size $n=30$ was taken from the lognormal distribution shown in Figure 3. For each sample, bootstrap CIs were computed using 5,000 bootstrap samples, for four quantities: the mean $(\mathbf{M})$, the $20 \%$ trimmed mean (TM), the median (MD), and the Harrell-Davis estimate of the 50th quantile (HD). In each panel, the vertical black line indicates the population value. This figure is available as a separate pdf file. It was created using the $\mathrm{R}$ notebook coverage, which is available in pdf $\underline{\mathbf{h t m l}}$ and $\underline{. \boldsymbol{R m d}}$ formats. 


\section{Limitations of the bootstrap}

Before we look at examples of applications of the bootstrap, it is worth clarifying two common misconceptions. First, the bootstrap is sometimes presented as being robust, but this is not the case. There are several statistical definitions of robustness, but loosely speaking, one important aspect is whether one extreme value can influence the outcome of a statistical test, in particular a confidence interval (Wilcox, 2017). Let's look at the example in Figure 6. We consider a single sample $(n=11)$ in which the largest value is progressively increased. As a result, the sample mean increases progressively, because it can be influenced by a single extreme value (Figure 6A). Similarly, the standard T-test CI for the mean also increases, because it relies on the variance, which is a non-robust measure of spread. If instead we use the bootstrap, the CIs are also inflated by the extreme value (Figure 6B). However, because the bootstrap accommodates asymmetric CIs, the effect is only seen on the side of the extreme value. So, although the bootstrap CI of the mean is not robust, it is in this case more informative than the standard one. Finally, if we use the bootstrap to make inferences about the median, the CIs are not affected at all by the extreme value (Figure 6C) because the median (and not the bootstrap procedure) is a robust estimator of location. 

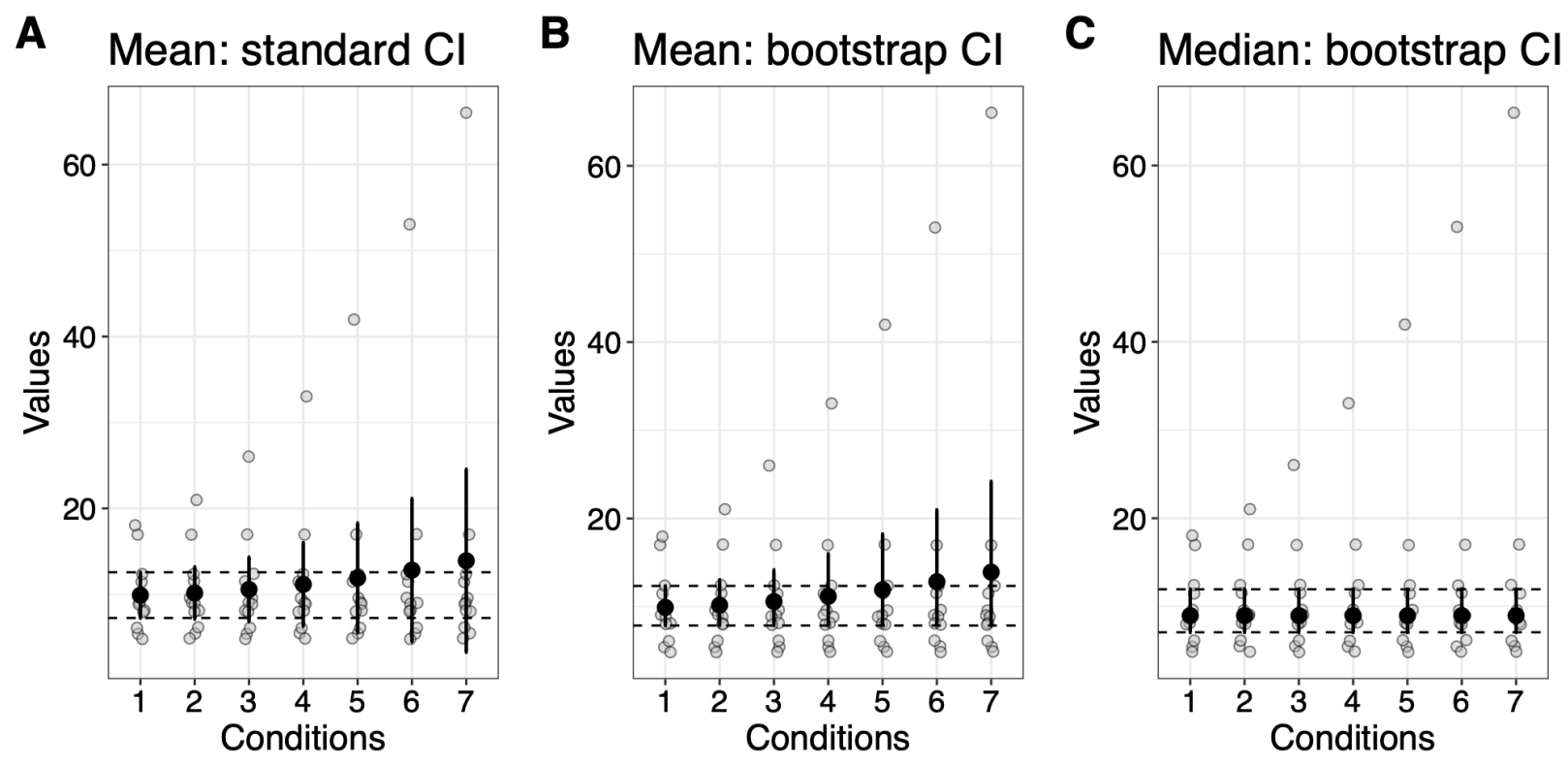

Figure 6. The bootstrap is not robust. Grey disks are observations. In each condition we consider a sample of $n=11$ observations. The 10 lowest observations are constant across conditions and panels. The largest observation is progressively shifted upward from condition 1 to 7 . In each condition, the black disk marks the sample estimate (mean or median), and the vertical line marks the bounds of the CI. For ease of comparison, the horizontal dashed lines indicate the bounds of the CI in condition 1. A. Standard T-test CI for the mean. B. Bootstrap CI for the mean. C. Bootstrap CI for the median. All bootstrap CI were computed using 2,000 bootstrap samples. This figure is available as a separate pdf file. It was created using the $\mathrm{R}$ notebook notrobust, which is available in $\mathbf{p d f}, \underline{\mathbf{h t m l}}$ and $\underline{\boldsymbol{R} \boldsymbol{m \boldsymbol { d }}}$ formats.

In sum, Figure 6 helps illustrate an important lesson: robustness comes from a conjunction of choices - a method to build a confidence interval, and the estimator for which a confidence interval is built. And unfortunately there is no universal solution. Depending on the types of distributions considered, different methods offer different performances and help answer different questions (Wilcox, 2017).

Second, the bootstrap is sometimes presented as not making any inferences about the data, unlike standard parametric methods. Although the bootstrap does not make assumptions about the shape of the distribution (the shape is not defined by parameters, hence the non-parametric label), it does make very strong assumptions about the data: indeed the bootstrap assumes that the observations in the original sample are the only ones that can ever be observed! This strong assumption explains why the bootstrap does not work well with small sample sizes - in some 
situations it gives weirdly shaped bootstrap distributions and inaccurate CIs. This is the case for instance when making inferences about the median, as we will see in a later example. That's because the median, as well as most quantile estimators, do not deal well with tied values, which tend to occur frequently in bootstrap samples derived from small original samples. Several solutions exist: for instance to use estimators that can handle tied values, such as the HarrellDavis quantile estimator (Harrell \& Davis, 1982), or to use a bootstrap technique that makes parametric assumptions, such as the percentile-t technique - see description later in the tutorial. More importantly, like any other statistical method, the bootstrap has no magical property: it is only useful when a sufficient sample size has been collected. Which brings us to an important question: how do we know that a method does what we expect it to do? Using simulations of course!

\section{Using simulations to check the behaviour of bootstrap confidence intervals}

As we mentioned previously, the behaviour of confidence intervals can only be defined in the long-run. There is no guarantee for a single experiment. This was illustrated in Figure 5, in which 50 simulated experiments were carried out, each time drawing a sample of size $\mathrm{n}=30$, and for each sample a bootstrap CI was computed for each of four measures of central tendency: the mean, the $20 \%$ trimmed mean, the median, and the Harrell-Davis estimator of the 50th quantile. Each of these estimators can be used to quantify the location of the bulk of the observations, although they behave differently depending on skewness, sample size and in the presence of outliers (Wilcox, 2017). They also answer slightly different questions about the data: for instance, when we use the $20 \%$ trimmed mean, we make inferences about the $20 \%$ trimmed mean of the population, not another quantity.

In our example, across simulated experiments, it is striking to see the large changes in position and width of the CIs: sometimes they include the population value, sometimes they don't, and they can be narrow or wide. This is a healthy reminder that inferences from a single experiment should be considered with caution. 
More generally, to assess CI methods, we proceed by performing simulations in tightly controlled conditions, for which we know what the result is. For instance, in Figure 4, we sampled from the lognormal population illustrated in Figure 3. Having defined a population of interest, we draw random samples of a certain size, and for each sample calculate a quantity of interest (say the median) and its confidence interval. In this approach, we can vary any aspect or parameter of the simulation we want to investigate: the population we sample from, the size of the sample, the quantity to estimate, the method used to build the CI, and for bootstrap methods, the number of bootstrap samples.

Then, what do we look for? For CIs, the first concern is coverage. For a 95\% CI, we need to ensure that in the long-run, 95\% of such CIs do contain the population value we try to estimate. For instance, Figure 7A shows the results of a simulation in which in each iteration, we took a sample of size $n=10$ and computed bootstrap CIs for the same four measures of central tendency we used in Figure 5. We varied the number of bootstrap samples from 500 to 10,000, in steps of 500. The results confirm the observations we made in Figure 6: bootstrap CIs of the mean do not perform well, with coverage near $81 \%$. Increasing sample size to $n=30$ improves matters, but still leads to coverage of about $88.5 \%$, instead of the expected $95 \%$ (Figure $7 \mathrm{~B}$ ). So using the mean in conjunction with the bootstrap is clearly not recommended. Other measures of central tendency are associated with CI coverage much closer to the nominal level. 

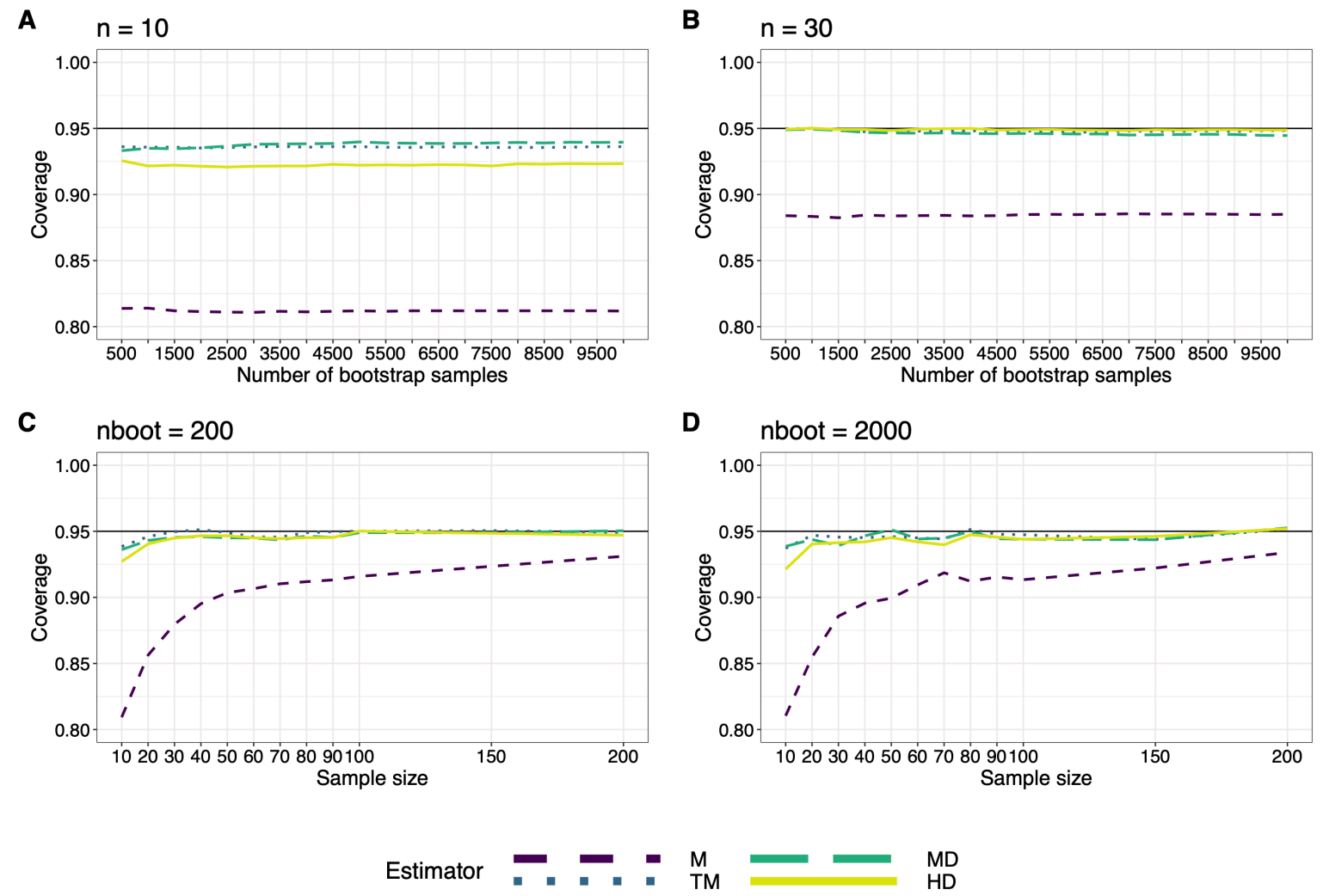

Figure 7. Confidence interval coverage. Results of simulations in which we sampled from the standard lognormal distribution illustrated in Figure 3. For each sample, bootstrap CIs were computed for four quantities: the mean (M), the 20\% trimmed mean (TM), the median (MD), and the Harrell-Davis estimate of the 50th quantile (HD). For each combination of sample size and number of bootstrap samples, coverage was computed as the proportion of simulations in which the CI included the population value. The number of iterations per simulation was 5,000 for panels A, B and D, and 10,000 for panel C. nboot $=$ number of bootstrap samples. This figure is available as a separate pdf file. It was created using the $\mathrm{R}$ notebook coverage, which is available in $\mathbf{p d f}, \underline{\mathbf{h t m l}}$ and $\underline{\mathbf{R m \boldsymbol { d }}}$ formats.

Instead of varying the number of bootstrap samples, we could also choose a fix number, and vary the sample size. Whether we used 200 or 2000 bootstrap samples, the main determinant of coverage was sample size (Figure 7C-D). Again bootstrap CIs for the mean performed poorly.

The results from Figure 7 suggest that the number of bootstrap samples does not affect the coverage of the CIs. However, this result cannot be generalised to all situations. And the number of bootstrap samples affects other aspects of the results, such as the precision of the bootstrap $P$ values and the width of the CIs (see extra figures in the notebook coverage.Rmd). For instance, 
given two methods with appropriate coverage, the one that tends to give shorter CIs seems

preferable, as it reduces our uncertainty about the population value in the long-run. So there are various ways to decide on the number of bootstrap samples to use, and not surprisingly, there is a whole statistical literature on the topic (Davidson \& MacKinnon, 2000; Hesterberg, 2015; Olive, 2014 chap. 9; Racine \& MacKinnon, 2007a, 2007b). In our experience, using 1,000 bootstrap samples appears to be sufficient if the goal is to have coverage at the nominal level. For statistical power, there might be situations where more samples are required. Given the speed of modern computers, for relatively simple applications there is no reason not to use 5,000 or even 10,000 bootstrap samples - except extra time and energy consumption, there is no negative effect of using more samples.

The choice of the number of bootstrap samples for a particular type of data and analysis is best made using simulations, as demonstrated in Figure 7 for instance. Another approach is to run the same bootstrap analysis a few times: the results should not change much across analyses of the same data. One aspect of the results to consider is the stability of the CI bounds. Let say we perform one experiment and obtain the sample in Figure 8A. We could then compute CIs for different measures of central tendency (Figure 8B). But what happens if we compute a bootstrap CI several times using the same data? Because of random sampling, the results differ slightly across CIs (Figure 8C). And this variability decreases with the number of bootstrap samples (Figure 8D). In this example, there is also more variability for the higher bound of the CI than the lower bound, which is explained by the skewness of the original sample. So the number of bootstrap samples should also be chosen based on the level of precision desired and the context in which the results are interpreted (Hesterberg, 2015). To obtain stable CI bounds, Hesterberg (2015) recommends at least 10,000 in routine applications, and much more when the outcome of the test is used to make strong decisions. For complex applications, for instance brain imaging analyses, such choices could lead to extra minutes, hours and sometimes days of calculations, but this extra time remains short relative to the time spent acquiring the data. 
A

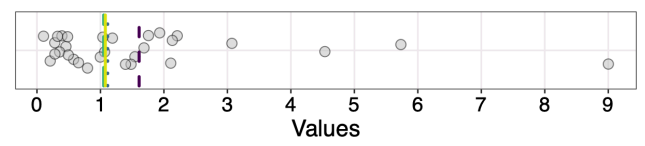

Estimator - - $=\mathrm{TM}_{\mathrm{M}}^{\mathrm{M}} \mathrm{MD}$

B

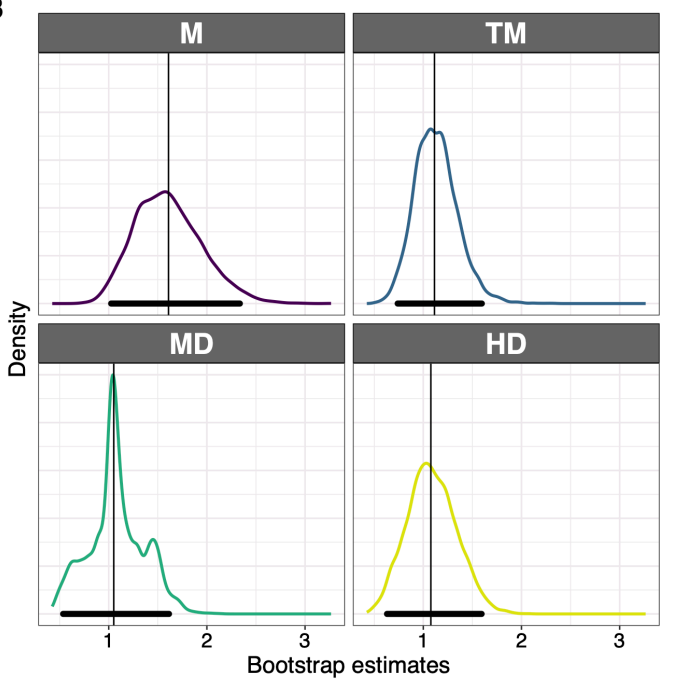

C

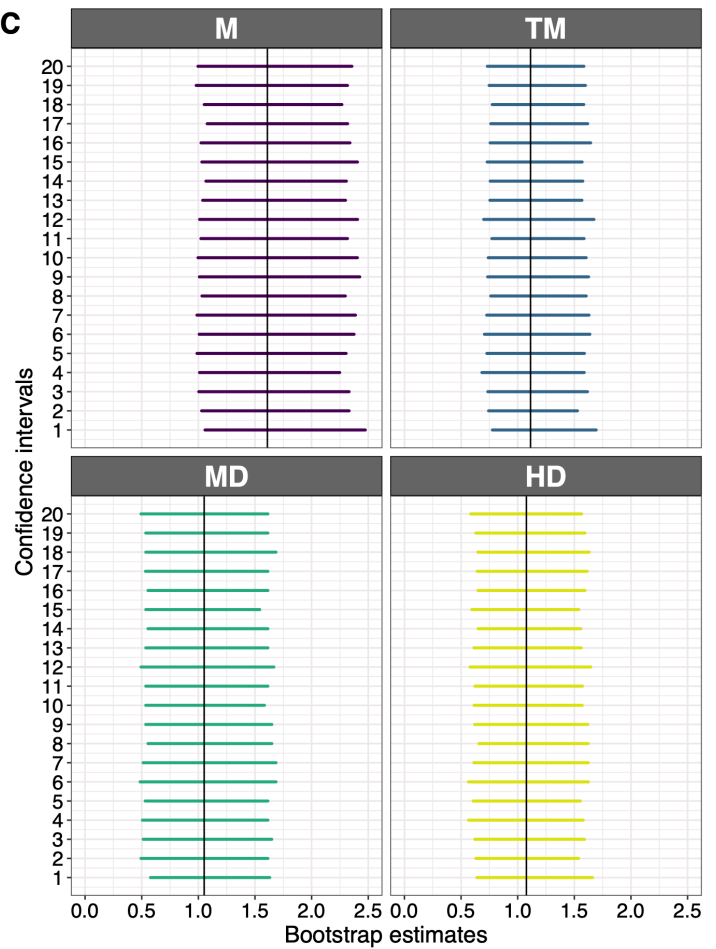

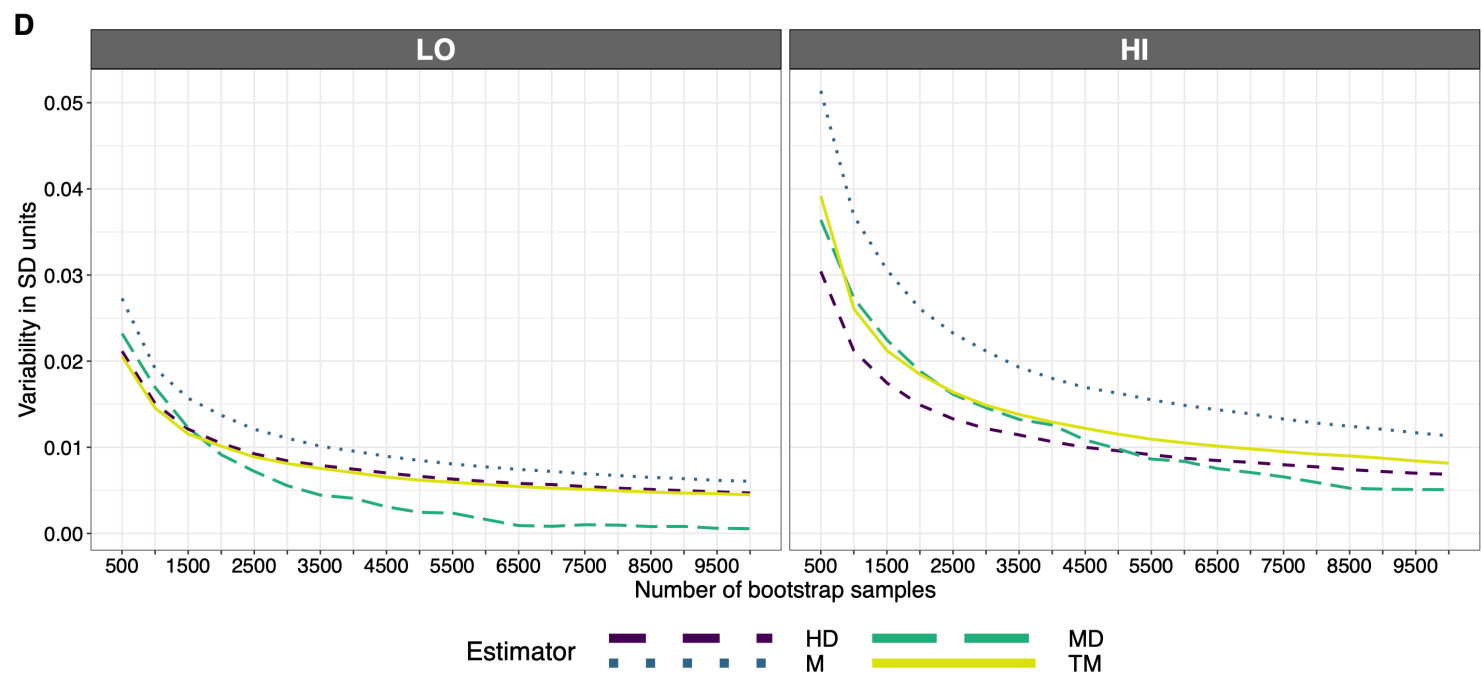

Figure 8. Performance diagnostics: stability. A. One sample $(n=30)$ from a standard lognormal distribution. B. Four bootstrap sampling distributions and CIs computed using 5,000 bootstrap samples, for four quantities: the mean (M), the $20 \%$ trimmed mean (TM), the median (MD), and the Harrell-Davis estimate of the 50th quantile (HD). C. Twenty bootstrap CIs for the same sample illustrated in panel A. 500 bootstrap samples were used to compute each CI. D. Variability of the CI bounds, measured as the standard deviation across 2,000 CI bounds, as a function of the number of bootstrap samples. This figure is available as a separate pdf file. It was created using the $\mathrm{R}$ notebook coverage, which is available in pdf, $\underline{\text { html }}$ and. $\mathbf{R m d}$ formats. 


\section{Group comparisons}

The examples covered so far used one-sample CIs. We now turn to the topic of group comparisons. Similarly to the one-sample case, the bootstrap for group comparisons follows the logic of the data acquisition process. For two independent groups, we sample observations with replacement independently from each group. If group 1 has $\mathrm{n} 1$ observations, we sample $\mathrm{n}$ observations with replacement. Similarly, in group 2 we sample n2 observations with replacement. If the groups differ in sample size, we preserve this size difference in our bootstrap samples.

Now let say we conducted an experiment and collected observations from two groups, each from a different population (Figure 9A \& B). Because of previous research, we suspect that the groups might differ in their right tails (more extreme observations). So instead of the traditional measures of central tendency, like the mean or the median, here we estimate the 3rd quartile of the marginal distributions. Then we use the bootstrap to derive a CI for the 3rd quartile of each group, as well as for the difference between groups. Twenty bootstrap samples are illustrated in Figure 9C. For each bootstrap sample, we compute the difference between the two bootstrap estimates; or some other quantity of interest: it could be some transformation of the difference, the ratio between groups, etc. Here, to illustrate that with the bootstrap we can build confidence intervals for any quantity, the group estimation is performed on a normalised difference of quartiles, defined as (q3gp1- q3gp2) / (q3gp1+ q3gp2), thus accounting for the total magnitude. The CI for the normalised difference is compatible with a value of zero, but also more negative values. The CI actually contains the population normalised difference (-0.17), but many more trials would be required to get a narrower CI that excludes zero. For instance, with a random sample of 200 observations for each group we obtained a CI of [-0.22, -0.11]. Of course, one could perform a simulation in which sample size is varied systematically, to determine the number of observations needed to detect or precisely estimate the effect. 
A
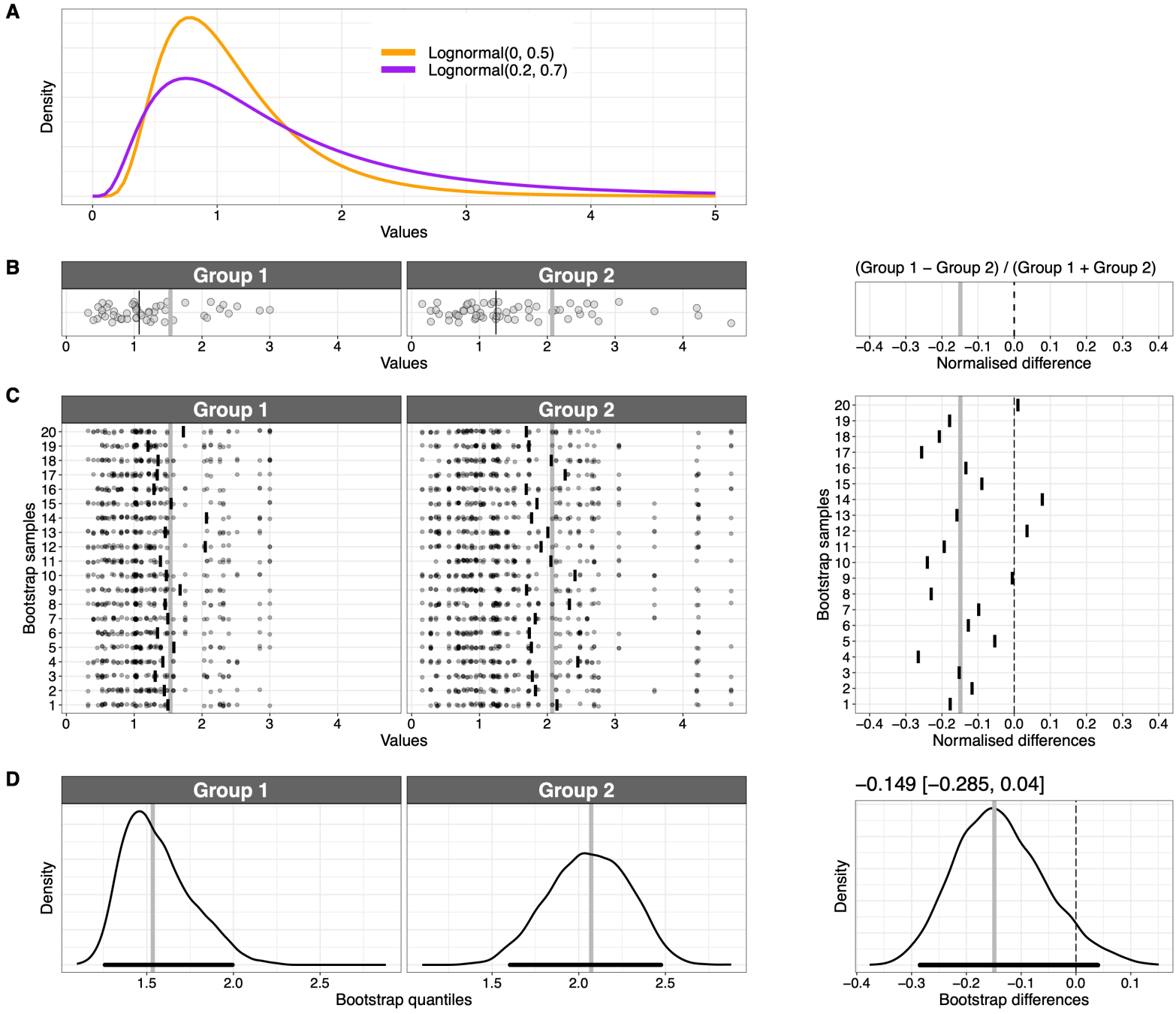

Figure 9. Comparison of two independent groups. A. Two populations that differ in skewness. B. Two independent samples, one from each population. Group 1 contains $n=50$ observations (left panel); group 2 contains $n=60$ observations (middle panel). The thick vertical grey line marks the 3rd quartile; the thin vertical black line marks the 2nd quartile (median). In the right panel, the thick vertical grey line marks the sample normalised group difference between 3rd quartiles, whereas the thin vertical black dashed line is a zero reference line. The same lines appear in subsequent panels. C. Examples of bootstrap samples. Each disk is a bootstrap observation. For each bootstrap sample the short vertical black line indicates the bootstrap 3rd quartile (left and middle panels), or the normalised difference (right panel). D. Bootstrap distributions of 3rd quartiles for the two groups (left and middle panels) and of the normalised quartile difference (right panel). The thick horizontal black lines indicate $95 \%$ bootstrap CIs. This figure is available as a separate pdf file. It was created using the $\mathrm{R}$ notebook $2 \mathrm{indgps}$, which is available in pdf,$\underline{\mathbf{h t m l}}$ and $\underline{. \boldsymbol{R} \boldsymbol{m} \boldsymbol{d}}$ formats. 


\section{Correlations}

In addition to group comparisons, another common topic in psychology is to make inferences about correlations. To compute bootstrap CI for correlations, the basic recipe is to sample pairs of observations with replacement. Again, we follow the data acquisition process: if different measurements were made in the same participants, then participants should be sampled with replacement, keeping all their measurements together. The same strategy applies to regression coefficients. Correlation and linear regression are huge topics, so we only address basic principles here (Wilcox, 2017). In general, for robust estimators, the bootstrap performs well, meaning that CIs with the expected coverage are obtained. In contrast, Pearson's correlation and the standard ordinary least square (OLS) regression are problematic for several reasons. Satisfactory bootstrap CIs can nevertheless be obtained with a simple adjustment to the way the quantiles of the bootstrap distributions are computed - see details in (Wilcox, 2009, 2017). However, estimators robust to univariate, or even better multivariate, outliers should be preferred to these standard yet outdated methods (e.g. Pernet, Wilcox, \& Rousselet, 2013).

Figure 10 presents an application of the bootstrap method to correlation analyses. Two measurements were made in two independent groups of participants (both $n=50$ ). Dependent cases are covered in the notebook compcorr.Rmd - see also Wilcox (2016, 2017). Group 1 was sampled from a non-normal population with Spearman's correlation 0.5, group 2 from a nonnormal population with Spearman's correlation 0.6. Spearman’s correlation quantifies monotonic relationships and is robust to univariate outliers (Pernet et al., 2013). We compute a bootstrap CI for each correlation by sampling pairs of observations with replacement, independently in each group.

In our experience, often two correlations are presented side-by-side, with the implicit assumption that if one is significant according to some arbitrary $P$ value threshold, and the other is not, then the two correlations differ. This is however a classic interaction fallacy, akin to looking at the simple effects in an ANOVA, without testing the interaction (Gelman \& Stern, 2006;

Nieuwenhuis et al., 2011). So an explicit comparison of correlation coefficients is needed. Unfortunately, the popular comparison of correlation coefficients using Fisher's $z$ transform is inappropriate because it is not robust to deviation from normality (Duncan \& Layard, 1973). In 
contrast, the comparison of two correlation coefficients is straightforward with the bootstrap. To compare two independent correlation coefficients using the bootstrap, we proceed like this:

- $\quad$ sample participants with replacement, independently in each group (concretely, for 2 groups, we sample dyads of observations, preserving the dependency among observations);

- compute the two correlation coefficients based on the bootstrap samples;

- $\quad$ save the difference between correlations;

- execute the previous steps many times;

- $\quad$ use the distribution of bootstrap differences to derive a confidence interval. 
A
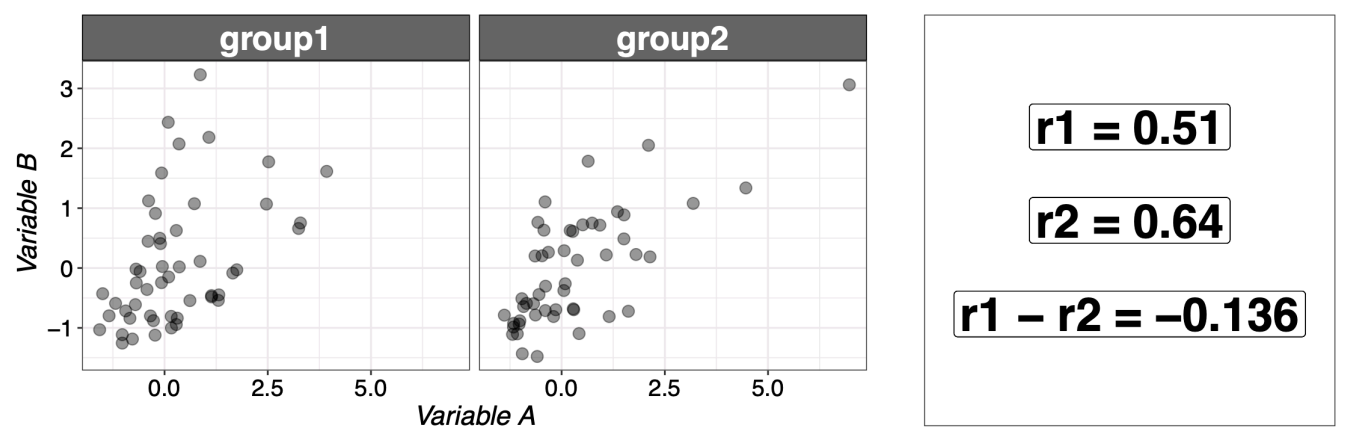

B
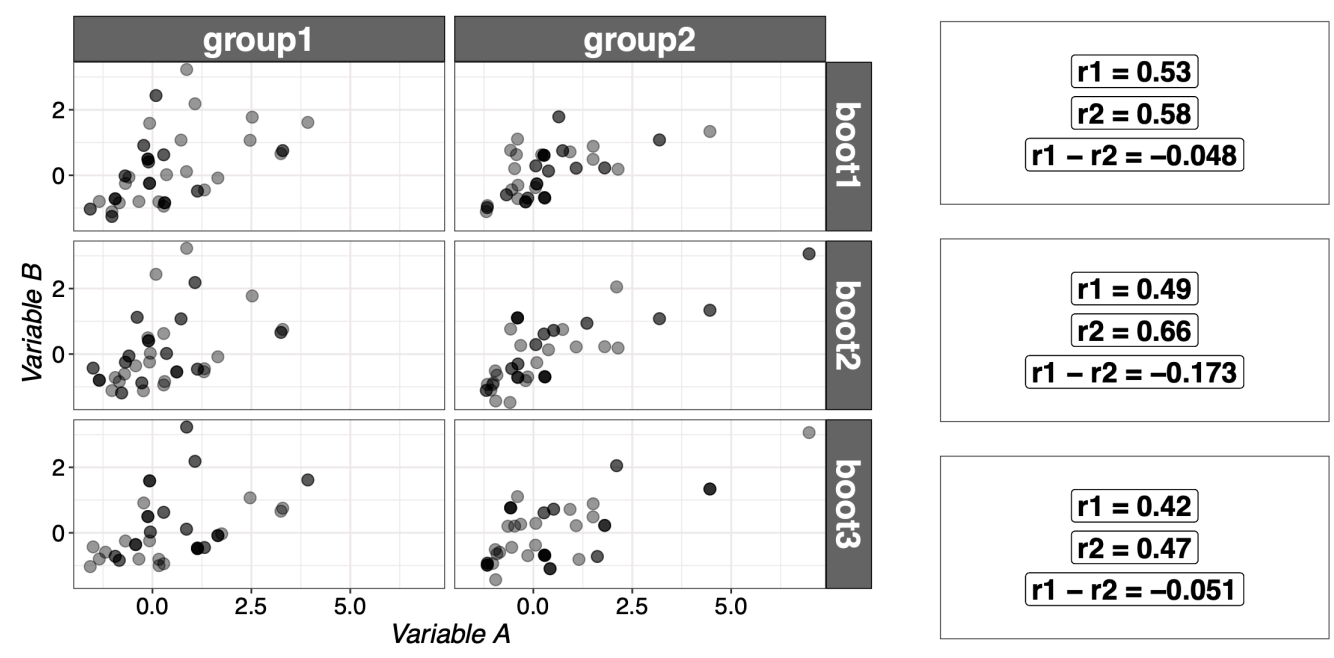

C
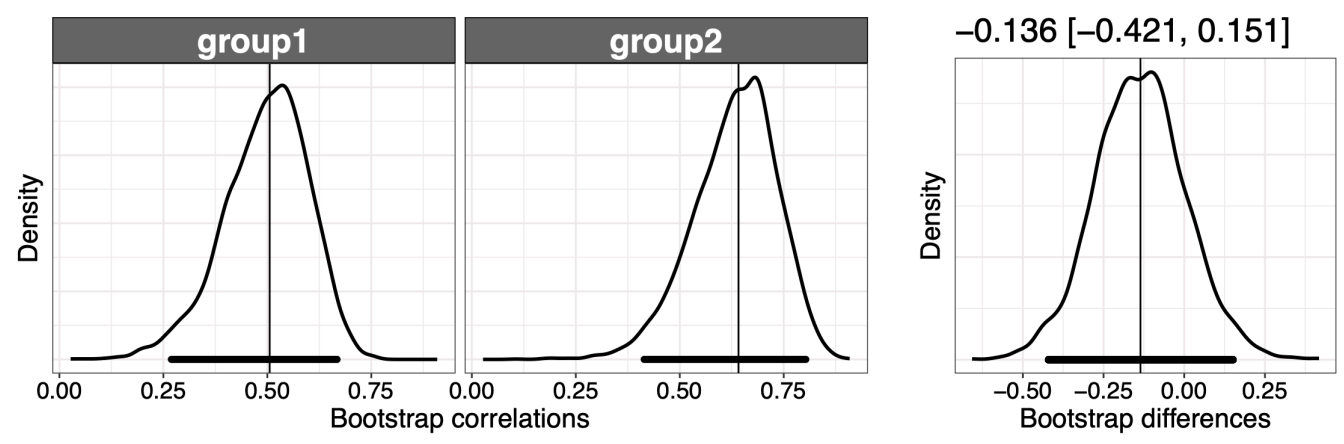

Figure 10. Bootstrap correlation analysis. A. Independent correlations, one in each of two groups. Sample size is $n=50$ in each group. Data from group 1 are from a population with correlation 0.5. Data from group 2 are from a population with correlation 0.6. The panel on the right reports the Spearman's correlation coefficient for each group and their difference. B. Examples of three bootstrap samples, one for each group, with the respective correlation coefficients in the right column. Darker disks represent observations sampled more than once. C. Bootstrap sampling distributions of the correlation coefficients and their differences, based on 5,000 bootstrap samples. The vertical lines mark the sample coefficients. The larger variability in group 1 leads to a broader bootstrap distribution. The thick horizontal black lines indicate $95 \%$ bootstrap CIs. This figure is available as a separate pdf file. It was created using the $\mathrm{R}$ notebook compcorr, which is available in pdf $\underline{\mathbf{h t m l}}$ and $\underline{\underline{R} \boldsymbol{m d}}$ formats. 
Based on 5,000 bootstrap samples, the bootstrap CI for the difference between Spearman's correlation coefficients suggests considerable uncertainty [-0.334, 0.24]: it is compatible with a population correlation difference of zero, as well as many positive and predominantly negative values. Larger sample sizes would be needed to detect and precisely estimate the population difference, as demonstrated by the simulation in the $\mathrm{R}$ notebook compcorr.Rmd. According to this simulation with 5,000 iterations (Figure 11A), 599 bootstrap samples and assuming non-normal distributions and a population difference of 0.1 (Spearman's correlation of 0.5 in group 1 and 0.6 in group 2), 420 observations are required in each group to achieve $50 \%$ of correct detection in the long run (true positives, or power). For 70\% correct detection, we need at least 665 observations in each group! Extra information can be provided by considering the shape of the sampling distributions. For instance, given 420 observations, in the long-run about $68.4 \%$ of experiments will provide difference estimates that are within +/- 0.05 of the population difference; with 665, the proportion is 78.9 (see the estimation precision curves in the $R$ notebook compcorr.Rmd). This type of information could be useful to plan a test of equivalence for instance (Campbell \& Gustafson, 2021; Kruschke, 2018; Lakens et al., 2018). Now, let us imagine that one population has a Spearman's correlation of zero and the other of 0.4, leading to a large population difference of -0.4 . In this situation, our simulation suggests that to reach $70 \%$ power, 77 observations are required per group; for 90\% power we need 128 observations. With these sample sizes, we can expect $48.3 \%(n=77)$ and $59.9 \%(n=128)$ of experiments to produce estimates within +/- 10 of the population difference. Thus, to successfully detect or estimate correlation differences between independent groups may require a lot more observations than is typically used in psychology experiments. 

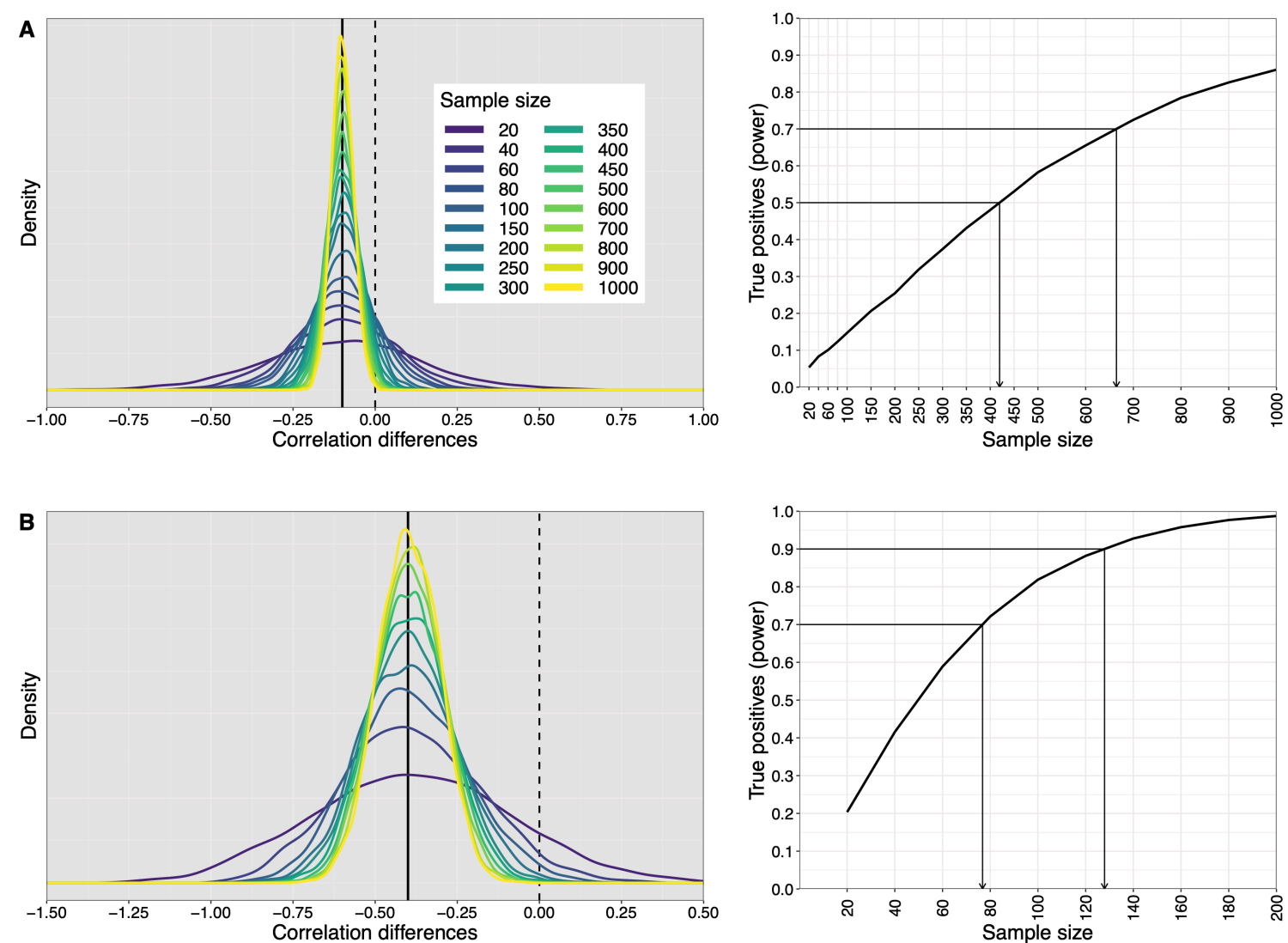

Figure 11. Simulations of correlation comparisons. A. One population had a Spearman's correlation of 0.5 and the other of 0.5 , leading to a population difference of -0.1 . The left panel shows sampling distributions of correlation differences from 5,000 simulation iterations. The vertical continuous black line marks the population correlation difference. The right panel shows a power curve. For each sample size along the $\mathrm{x}$-axis, the $\mathrm{y}$-axis indicates the proportion of simulation iterations that resulted in a $\mathrm{p}$ value $<$ 0.05 (or equivalently a 95\% confidence interval excluding zero), based on 599 bootstrap samples. The two arrows indicate the sample sizes required to reach two arbitrary levels of power $(50 \%$ and $70 \%)$. B. Same as A, except that one population had a Spearman's correlation of zero and the other a correlation of 0.4, leading to a population difference of -0.4 . In the right panel, the arrows point to sample sizes required to reach $70 \%$ and $90 \%$ power. This figure is available as a separate pdf file. It was created using the $\mathrm{R}$ notebook compcorr, which is available in pdf, $\mathbf{h t m l}$ and. $\mathbf{R m d}$ formats. 


\section{Extension to other designs}

The bootstrap can be extended beyond the simple comparisons of two groups we have covered so far: examples include multiple linear contrasts and various types of ANOVA designs, including ANCOVAs (Wilcox, 2017). Here we look at an application of the bootstrap to a hierarchical dataset. Imagine we collected reaction times from 20 participants in a lexical decision task, with 200 trials per condition (Word and Non-Word conditions, Figure 12). This example dataset was subsampled from a much larger dataset (Ferrand et al., 2010). The structure of the data is hierarchical because trials are nested within participants. There are many ways to analyse such data, but a substandard yet typical approach is to ignore the variability across trials by summarising the distribution for each participant and each condition using a single value (Rousselet \& Wilcox, 2019). For instance, we could summarise each of the 40 distributions in Figure $12 \mathrm{~A}$ using the $20 \%$ trimmed mean; we're then left with 20 values per condition and their differences (Non-Word minus Word, Figure 12B). In turn, we summarise the distributions across participants, for each condition and their difference: for simplicity here, we use again the 20\% trimmed mean. Finally, we use the bootstrap to compute CIs for the group trimmed means in each condition, and their difference (Figure 12G). To apply the standard bootstrap, we proceed as we did for the correlations: we sample participants with replacement, that is pairs of trimmed means, one in each condition. This approach ignores, however, the variability across trials.

A hierarchical bootstrap can be used to exploit within and between subject variability. There are several approaches to implement a hierarchical bootstrap (Roberts \& Fan, 2004). One approach is to follow the data acquisition process by resampling first at the highest level (participants), then at the next level (trials). This is also called a nested bootstrap, because the trials are nested within participants. In each bootstrap sample, we sample participants with replacement; then for each participant, we sample trials with replacement, independently for each condition. The 20\% trimmed mean is computed for each condition across trials, and the results from the two conditions subtracted (Non-Word minus Word). Then, the 20\% trimmed mean is computed across participants for each condition and their difference. In our example, the bootstrap sampling distributions and the CIs from the hierarchical procedure are very similar to the standard bootstrap ones. However, this is not always the case. In particular, the hierarchical 
procedure can make substantial improvements when making inferences about quantiles or the median for small sample sizes. For instance, in Figure 12D, the bootstrap sampling distributions for the median are very irregular when using the standard bootstrap (sampling at the participant level only). This is because for small sample sizes, bootstrap samples tend to contain too many tied values, such that the bootstrap median takes only a few discrete values. With the nested bootstrap, sampling trials with replacement adds variability to the values from each participant, which ultimately leads to smoother bootstrap distributions and, in this example, slightly different CIs. 
A

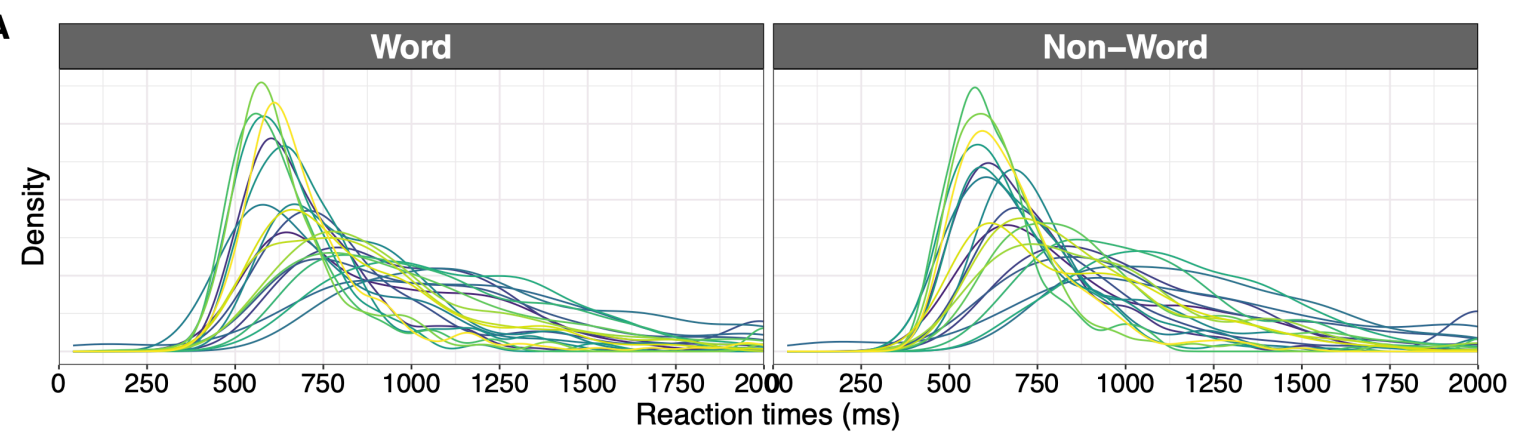

B

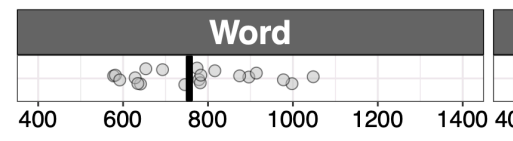

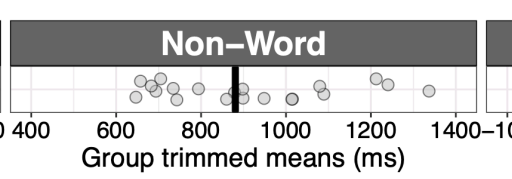
Group trimmed means (ms)

C
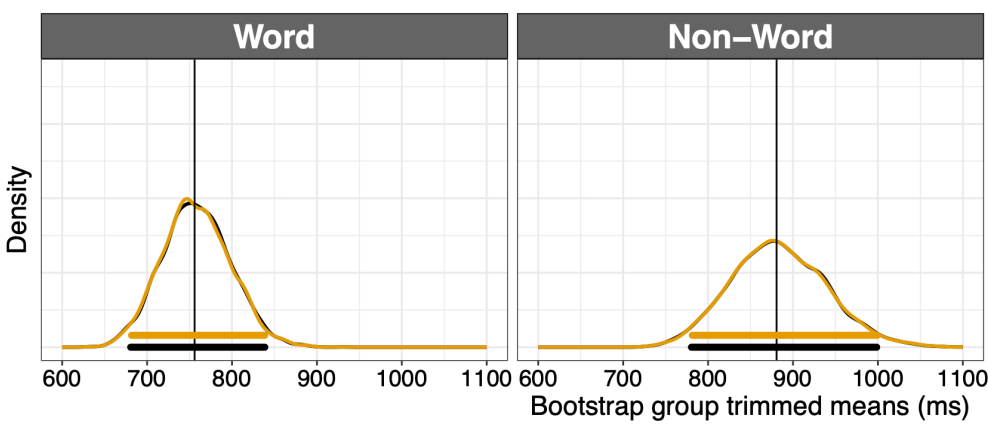

Non-Word
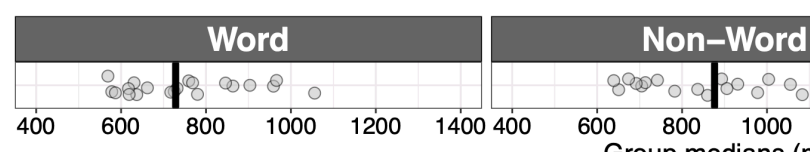

E
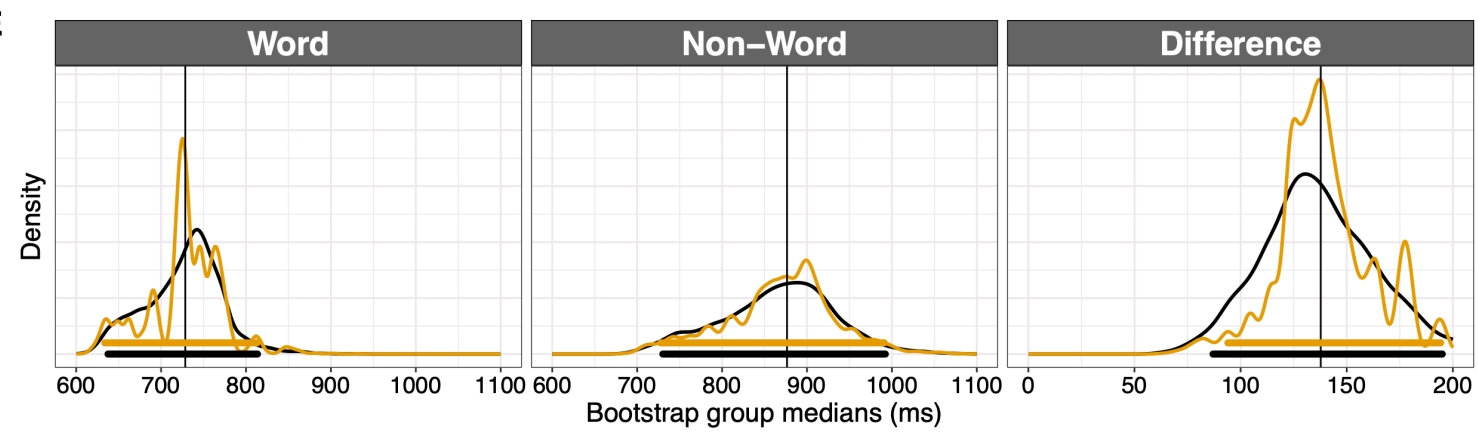

type

Hierarchical Non-hierarchical

Figure 12. Hierarchical bootstrap. A. Distributions of 200 reaction times in the Word and NonWord conditions for 20 participants. B. Distributions of $20 \%$ trimmed means across trials for the Word and Non-Word conditions and their differences. In each panel, there is a disk for each of the 20 participants. The vertical black lines mark the 20\% trimmed means across participants in each condition and the difference. C. Bootstrap distributions of $20 \%$ trimmed means across participants, based on 5,000 bootstrap samples. The thick horizontal lines indicate 95\% bootstrap CIs. Orange $=$ bootstrap at the participant level only, black $=$ hierarchical bootstrap of participants and their nested trials. $\mathbf{D}$. 
Distributions of participants' median reaction times in the two conditions and their differences. The vertical black lines mark the medians across participants. E. Bootstrap distributions of group medians. This figure is available as a separate pdf file. It was created using the $\mathrm{R}$ notebook 2 depgps, which is available in pdf html and $\underline{\text {.Rmd }}$ formats.

\section{Bootstrap-t technique}

Before we conclude, in this last section we introduce an alternative to the standard (percentile) bootstrap presented so far. As mentioned in the introduction, there are many variants of the bootstrap, but in psychology, two methods cover most of the applications: the standard bootstrap and the bootstrap-t technique - also known as the percentile-t bootstrap or the studentized bootstrap (Efron \& Tibshirani, 1994; Wilcox, 2017). As we saw in previous examples, for inferences on the population mean, the standard $\mathcal{T}$ - test and the bootstrap can give unsatisfactory results when sampling from skewed distributions, especially when sample size is small. To illustrate the problem with the t-test, imagine that we sample from populations of increasing skewness (Figure 13A). Here we use the so-called $g$ $\mathcal{E} h$ distributions, in which parameter $g$ controls the skewness, and parameter $h$ controls the thickness of the tails - a normal distribution is obtained by setting $g=h=0$ (Hoaglin, 1985; Yan \& Genton, 2019). If we take many samples of size $n=30$ from these distributions, and for each sample we compute a $T$ value, using the population mean as the null value, we obtain progressively more negatively skewed $T$ value sampling distributions (Figure 13B). However, when we perform a $\mathcal{T}$-test, the $\mathcal{T}$ values are assumed to be symmetric, irrespective of sample size. This assumption leads to incorrect CIs. The idea behind the bootstrap-t technique is to use the bootstrap to compute a data-driven $T$ distribution. In the presence of skewness, this $T$ distribution could be skewed, as suggested by the data. Then, the appropriate quantile of the bootstrap $T$ distribution is plugged into the standard CI equation to obtain a parametric bootstrap CI. 

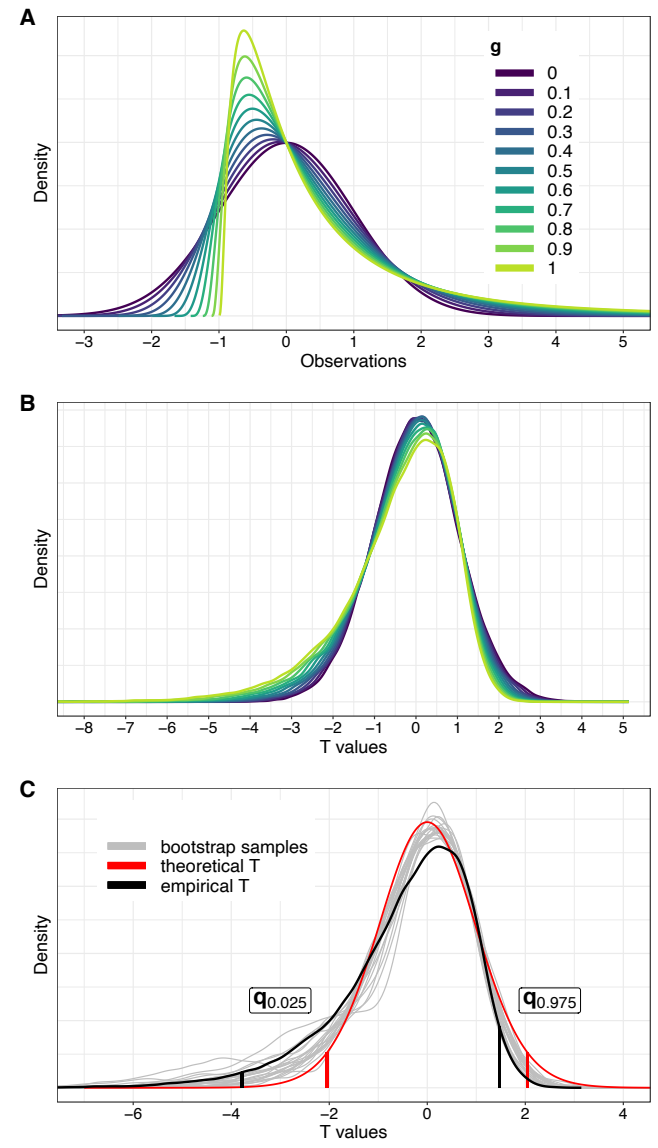
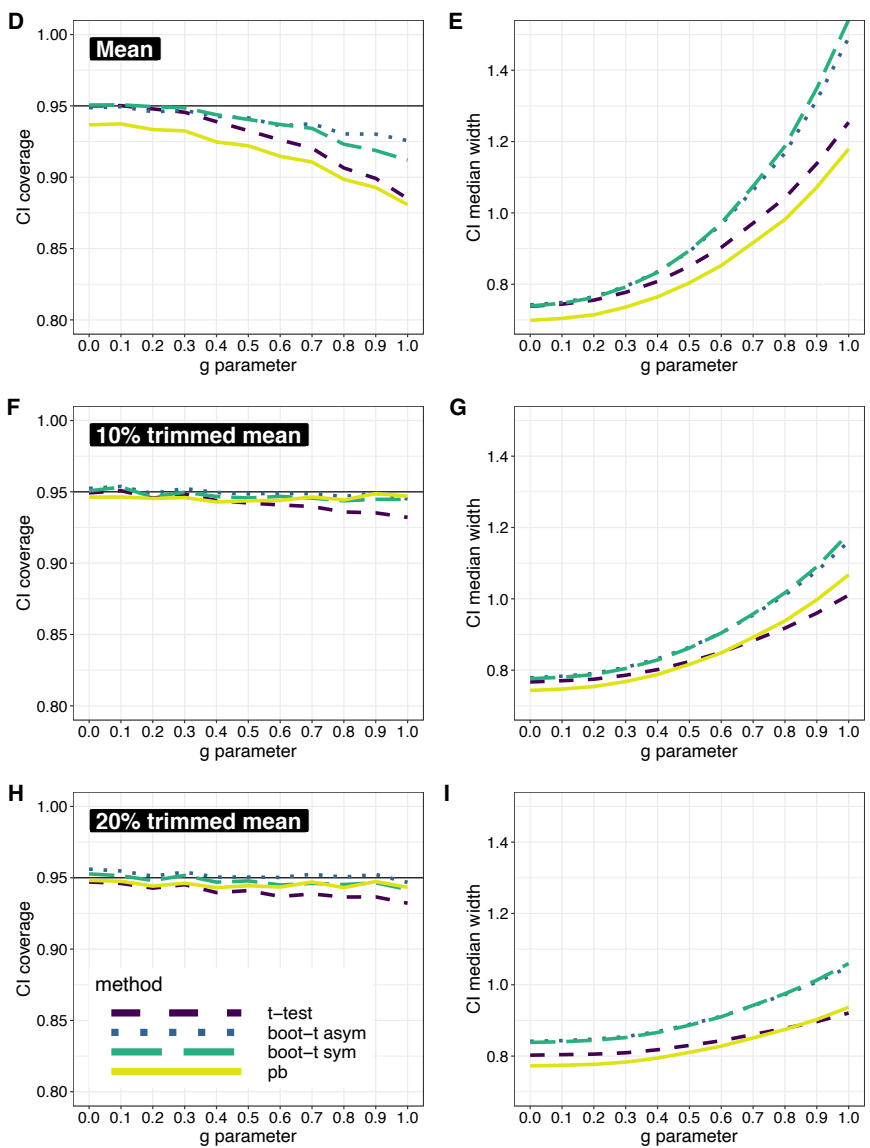

Figure 13. Bootstrap-t technique. A. Probability density functions for $g \in \mathcal{} h$ distributions. Parameter $g$ varies from 0 to 1 . Parameter $h=0$. B. Sampling distribution of $\mathcal{T}$ values for the different $g$ values. Results are based on a simulation with 50,000 iterations and samples of size $\mathrm{n}=30$. C. Comparison of $T$ distributions for $g=1$ : theoretical $T$ is the one used in the $T$ - test (red), empirical $T$ is from panel B (black). The red and black vertical lines indicate the $T$ quantiles for a $95 \%$ CI. The grey lines show examples of 20 bootstrap sampling distributions (each with 5,000 bootstrap samples), based on samples of size $\mathrm{n}=30$ from a $g \mathcal{E} h$ population with $g=1$ and $h=0$. D-I. Results of a simulation with 20,000 iterations, sample sizes of $\mathrm{n}=30$, and 599 bootstrap samples. Panels $\mathrm{D}$ and $\mathrm{E}$ show results for the mean, panels $\mathrm{F}$ and $\mathrm{G}$ for the $10 \%$ trimmed mean, and panels $\mathrm{H}$ and $\mathrm{I}$ for the $20 \%$ trimmed mean. This figure is available as a separate pdf file. It was created using the $\mathrm{R}$ notebook $p t b$, which is available in pdf, html and .Rmd formats.

Figure 14 illustrates the procedure to build a CI for the population mean (row A) and for the population $20 \%$ trimmed mean (row B). In both cases, we start with the same sample of 30 observations from a $g \mathscr{E}^{2} h$ distribution with $g=1$ and $h=0$. In a first step, we centre the distribution: for inferences on the mean, we subtract the mean from each observation in the 
sample, so that the mean of the centred distribution is now zero. This is a way to create a datadriven null distribution, in which there is no effect (the mean is zero), but the shape of the distribution and the absolute distance among observations are unaffected, as shown in the second column. For inferences on the $20 \%$ trimmed mean, we subtract the $20 \%$ trimmed mean from each observation, so that the centred distribution now has a 20\% trimmed mean of zero. In the next step, we sample with replacement from the centred distribution many times, and for each random sample we compute a $\mathcal{T}$ value. That way, we obtain a bootstrap distribution of $\mathcal{T}$ values expected by random sampling, under the hypothesis that the population has a mean (or 20\% trimmed mean) of zero, given the distribution of the data. Then, we use some quantile of the bootstrap $\mathcal{T}$ distribution in the standard CI equation. Because the bootstrap distribution is potentially asymmetric, we have two choices of quantiles: for a 95\% CI, either we use the 0.025 and the 0.975 quantiles of the signed $\mathcal{T}$ values to obtain a potentially asymmetric CI, also called an equal-tailed CI, or we use the 0.95 quantile of the absolute $T$ values, thus leading to a symmetric CI. In our example, for the mean the symmetric CI is [-0.34, 1.56] and the asymmetric CI is [0.07, 1.95]; for the $20 \%$ trimmed mean the symmetric CI is [-0.34, 0.57] and the asymmetric CI is [-0.28, 0.62]. So the choice of method can have a substantial impact on the CI. In general, when comparing 20\% trimmed means, there are indications that an asymmetric confidence interval is a bit better than a symmetric confidence interval. When comparing means, there are theoretical results suggesting that a symmetric confidence interval is preferable, but this issue is in need of further study (Wilcox, 2017). 
A
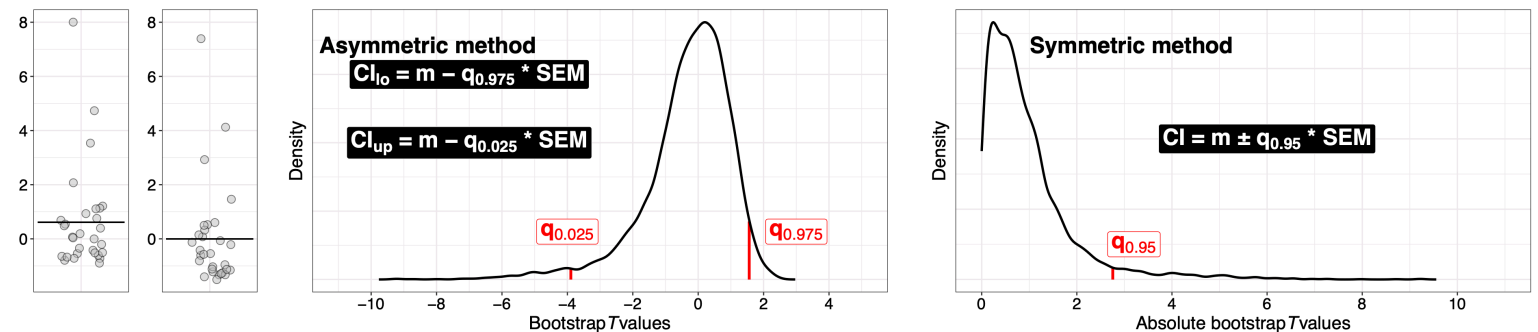

B
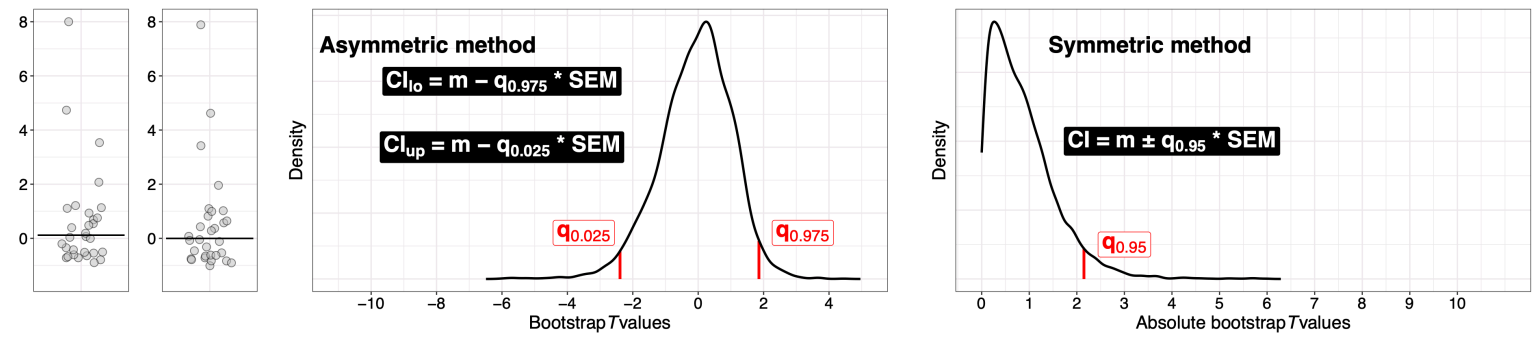

Figure 14. Bootstrap-t technique explained. A. Inferences on the population mean. The first panel illustrates a sample of size $\mathrm{n}=30$ from a $g$ $\mathcal{E} h$ distribution with $g=1$ and $h=0$. The same sample is used in rows $\mathrm{A}$ and $\mathrm{B}$. The horizontal line indicates the sample mean. In the second panel, the distribution has been mean centred, so that the sample mean is now zero. The third panel shows 5,000 bootstrap $T$ values obtained by sampling with replacement from the mean centred data. In the asymmetric bootstrap-t technique, the quantiles (red vertical lines) of that distribution of $\mathcal{T}$ values are used to define the CI bounds. The insets contain the formulas for the lower $\left(\mathrm{CI}_{\mathrm{lo}}\right)$ and upper bounds $\left(\mathrm{CI}_{\mathrm{up}}\right)$ of the $\mathrm{CI}$. Note that the lower $\mathcal{T}$ quantile is used to compute the upper bound (this not an error). In the symmetric bootstrap-t technique, one quantile of the distribution of absolute $T$ values is used to define the CI bounds. B. Same as A, but for inferences on the population $20 \%$ trimmed mean. This figure is available as a separate pdf file. It was created using the $\mathrm{R}$ notebook $p t b$, which is available in pdf $\underline{\mathbf{h t m l}}$ and $\underline{. \boldsymbol{R} \boldsymbol{m \boldsymbol { d }}}$ formats.

Why does this approach work better than the standard $T$ - test CI? Imagine we take multiple samples of size $\mathrm{n}=30$ from a $g \mathcal{E}^{2} h$ distribution with $g=1$ and $h=0$ (Figure $13 \mathrm{C}$ ). The standard $T$ test assumes the sampling distribution in red, symmetric around zero. From our simulation in Figure 13B, we know that the sampling distribution is actually asymmetric, with negative skewness, as shown in black. However, the black empirical distribution is unobservable, unless we can perform thousands of experiments. So, with the bootstrap, we try to estimate this correct, yet unobservable, sampling distribution. The grey curves in Figure 13C show examples of 20 simulated experiments: in each experiment, a sample of 30 observations is drawn, and then 5,000 bootstrap $\mathcal{T}$ values are computed by sampling with replacement from the 30 observations. The 
resulting bootstrap sampling distributions are negatively skewed and are much closer to the empirical distribution in black than the theoretical symmetric distribution in red. Thus, it seems that using data-driven $T$ distributions could help achieve better CIs than if we assumed symmetry.

How do these different methods perform? To find out we carry out simulations in which we draw samples from $g \mathcal{E} h$ distributions with the $g$ parameter varying from 0 to 1 , keeping $h=0$. For each sample, we compute a one-sample CI using the standard $\mathcal{T}$ - test, the two bootstrap-t methods just described, and the percentile bootstrap. When estimating the population mean, for all four methods, coverage goes down with skewness (Figure 13D). Among the parametric methods, the standard $T$ - test is the most affected by skewness, with coverage less than $90 \%$ for the most skewed condition. The asymmetric bootstrap-t CI seems to perform the best. The percentile bootstrap performs the worst in all situations, and has coverage systematically below $95 \%$, including for normal distributions.

In addition to coverage, it is useful to consider the width of the CIs from the different techniques (Figure 13E, G, I). The width of a CI is its upper bound minus its lower bound. For each combination of parameters, the results are summarised by the median width across simulations. At low levels of asymmetry, for which the three parametric methods have roughly $95 \%$ coverage, the CIs tend to be of similar widths. As asymmetry increases, all methods tend to produce larger CIs, but the $T$ - test produces CIs that are too short, a problem that stems from the symmetric theoretical $\mathcal{T}$ distribution, which assumes $\mathcal{T}$ values too small. Compared to the parametric approaches, the percentile bootstrap produces the shortest CIs for all $g$ values.

The low coverage observed in the previous simulations can be addressed by making inferences about trimmed means instead of the mean, which requires several adjustments to the $\mathcal{T}$-test equation (Tukey \& McLaughlin, 1963). With the 20\% trimmed mean, coverage improves for all methods, with values now close to the nominal level even for the most skewed distributions, though the $T$ - test performs less well than all three bootstrap techniques (Figure 13H). CIs are also overall shorter for all methods compared to CIs of the mean (Figure 13I). The results were similar when making inferences about the 10\% trimmed mean (Figure 13F-G). Also, the shorter CIs obtained with the percentile bootstrap compared to the bootstrap-t CIs, for similar coverage, 
suggest it could be beneficial to use the percentile bootstrap in conjunction with trimmed means. More generally, inferences about trimmed means are robust to outliers, which can have devastating effects on the power of methods using the mean (Wilcox, 2017; Wilcox \& Rousselet, 2018). Finally, based on simulations, it seems that to make inferences about the mean or when trimming less than 20\%, the bootstrap-t technique performs well in many situations and should be preferred over the percentile bootstrap; when trimming at least $20 \%$, the standard bootstrap approach is recommended (Wilcox, 2017).

\section{Conclusion}

In this tutorial, we introduced the bootstrap, its core mechanism, sampling with replacement, its main output, a bootstrap sampling distribution, and presented its strengths and weaknesses. The bootstrap is a very versatile approach to statistical inferences, but it is by no means a magical recipe. It can perform poorly in some situations, for instance when making inferences about means, or about medians for relatively small samples. But combined with robust estimators, the bootstrap often outperforms other approaches, and can provide a simple solution to compute CIs when analytical solutions do not exist: this is for instance the case when making inferences about certain quantile estimators, robust correlation and regression estimates, as well as other robust estimators not mentioned here (Wilcox, 2017). The bootstrap-t can be used to make inferences about means and trimmed means, and the approach can be extended to various ANOVA designs, improving statistical power in many situations relative to standard ANOVAs on means (Field \& Wilcox, 2017).

Thus, by learning the bootstrap, users do not only get a new powerful technique in their toolbox, but a whole set of techniques that can be applied to a large range of problems. The application of bootstrap techniques can bring potentially more informative answers relative to standard parametric approaches in psychology, which typically focus on mean only and are often inappropriate. For instance, reaction time distributions contain a lot of information that is thrown away by summarising them using the mean. The bootstrap, combined with quantile 
estimation, can help provide a much more detailed understanding of how such continuous distributions differ across conditions (Rousselet et al., 2017; Rousselet \& Wilcox, 2019).

This is not to imply that bootstrap approaches should be used to tackle any problem in psychological research. In a lot of situations, there are well-established and powerful methods that do not require the bootstrap: for instance diffusion models of reaction times (Matzke \& Wagenmakers, 2009; Voss et al., 2013), beta-binomial models of accuracy scores (Kruschke, 2014), and ordinal models of Likert scale results (Bürkner \& Vuorre, 2019; Liddell \& Kruschke, 2018).

Although the bootstrap might not be useful to all psychology researchers, learning about it has other important benefits. Because the percentile bootstrap can accommodate any estimator, it nudges users towards justifying their choice of estimators. In the special case of inferences about central tendency, users free of the standard $T$-test can choose from many more options than the mean (or trimmed means). The bootstrap also provides a gentle introduction to data-driven simulations. Once that concept is acquired, it becomes easier to learn how to perform simulations in general. The bootstrap also helps understand the key idea of a sampling distribution and it makes explicit the data acquisition process and the structure of the data, all without requiring mathematical expertise. Thus, the bootstrap should have an important part in a modern statistical curriculum, with a focus on simulations and the assessment of the performance of our statistical methods (Hesterberg, 2015; Steel et al., 2019; Tintle et al., 2015).

\section{Funding}

The authors declare no sources of funding for this work.

\section{Author Contributions}

G. A. Rousselet wrote the code for the simulations and the figures. R. R. Wilcox provided the functions implementing the bootstrap methods and the $g \mathcal{G} h$ distributions. C. R. Pernet verified 
the code. G. A. Rousselet wrote the first draft of the manuscript, and all authors critically edited it. All authors approved the final submitted version of the manuscript.

\section{Conflicts of Interest}

The authors declare that there were no conflicts of interest with respect to the authorship or the publication of this article, besides this article enhancing their CVs.

\section{References}

Bååth, R. (2015). The Non-parametric Bootstrap as a Bayesian Model-Publishable Stuff. Publishable Stuff. http://www.sumsar.net/blog/2015/04/the-non-parametric-bootstrapas-a-bayesian-model/

Bååth, R. (2018). beepr: Easily Play Notification Sounds on any Platform. https://CRAN.Rproject.org/package $=$ beepr

Bürkner, P.-C., \& Vuorre, M. (2019). Ordinal Regression Models in Psychology: A Tutorial. Advances in Methods and Practices in Psychological Science, 2(1), 77-101. https://doi.org/10.1177/2515245918823199

Campbell, H., \& Gustafson, P. (2021). Re:Linde et al. (2021): The Bayes factor, HDI-ROPE and frequentist equivalence tests can all be reverse engineered - almost exactly - from one another. arXiv. https://doi.org/10.48550/ARXIV.2104.07834 
Davidson, R., \& MacKinnon, J. G. (2000). Bootstrap tests: How many bootstraps? Econometric Reviews, 19(1), 55-68. https://doi.org/10.1080/07474930008800459

Duncan, G. T., \& Layard, M. W.J. (1973). A Monte-Carlo study of asymptotically robust tests for correlation coefficients. Biometrika, 60(3), 551-558. https://doi.org/10.1093/biomet/60.3.551

Efron, B. (1979). Bootstrap Methods: Another Look at the Jackknife. The Annals of Statistics, 7(1), 1-26. https://doi.org/10.1214/aos/1176344552

Efron, B., \& Hastie, T. (2016). Computer Age Statistical Inference. Cambridge University Press.

Efron, B., \& Tibshirani, R. (1986). Bootstrap Methods for Standard Errors, Confidence Intervals, and Other Measures of Statistical Accuracy. Statistical Science, 1(1), 54-75. https://doi.org/10.1214/ss/1177013815

Efron, B., \& Tibshirani, R. (1994). An Introduction to the Bootstrap. Chapman and Hall/CRC.

Field, A. P., \& Wilcox, R. R. (2017). Robust statistical methods: A primer for clinical psychology and experimental psychopathology researchers. Behaviour Research and Therapy, 98, 19-38. https://doi.org/10.1016/j.brat.2017.05.013

Gelman, A., \& Stern, H. (2006). The Difference Between "Significant" and "Not Significant" is not Itself Statistically Significant. The American Statistician, 60(4), 328-331. https://doi.org/10.1198/000313006X152649 
Goerg, G. M. (2011). Lambert W random variables - A new family of generalized skewed distributions with applications to risk estimation. Annals of Applied Statistics, 5(3), 21972230.

Goerg, G. M. (2022). LambertW: Probabilistic Models to Analyze and Gaussianize Heavy-Tailed, Skewed Data.

Greenland, S., Senn, S. J., Rothman, K. J., Carlin, J. B., Poole, C., Goodman, S. N., \& Altman, D. G. (2016). Statistical tests, P values, confidence intervals, and power: A guide to misinterpretations. European Fournal of Epidemiology, 31(4), 337-350. https://doi.org/10.1007/s10654-016-0149-3

Hankin, R. K. S. (2006). Special functions in R: introducing the gsl package. $R$ News, 6(4).

Harrell, F. E., \& Davis, G. E. (1982). A new distribution-free quantile estimator. Biometrika, 69(3), 635-640. https://doi.org/10.1093/biomet/69.3.635

Hasselman, B. (2022). nleqslv: Solve Systems of Nonlinear Equations. https:/ / GRAN.Rproject.org $/$ package $=$ nleqslv

Hesterberg, T. (2015). What Teachers Should Know About the Bootstrap: Resampling in the Undergraduate Statistics Curriculum. The American Statistician, 69(4), 37 1-386. https://doi.org/10.1080/00031305.2015.1089789

Hoaglin, D. C. (1985). Summarizing Shape Numerically: The g-and-h Distributions. In Exploring Data Tables, Trends, and Shapes (pp. 461-513). John Wiley \& Sons, Ltd. https://doi.org/10.1002/9781118150702.ch11 
Kruschke, J. K. (2014). Doing Bayesian Data Analysis (2nd Edition). Academic Press.

Kruschke, J. K. (2018). Rejecting or Accepting Parameter Values in Bayesian Estimation. Advances in Methods and Practices in Psychological Science, 1(2), 270-280. https://doi.org/10.1177/2515245918771304

Lakens, D., Scheel, A. M., \& Isager, P. M. (2018). Equivalence Testing for Psychological Research: A Tutorial. Advances in Methods and Practices in Psychological Science, 1(2), 259-269. https://doi.org/10.1177/2515245918770963

Liddell, T. M., \& Kruschke, J. K. (2018). Analyzing ordinal data with metric models: What could possibly go wrong? Fournal of Experimental Social Psychology, 79, 328-348. https://doi.org/10.1016/j.jesp.2018.08.009

Limpert, E., \& Stahel, W. A. (2017). The log-normal distribution. Significance, 14(1), 8-9. https://doi.org/10.1111/j.1740-9713.2017.00993.x

Limpert, E., Stahel, W. A., \& Abbt, M. (2001). Log-normal Distributions across the Sciences: Keys and Clues. BioScience, 51(5), 341-352. https://doi.org/10.1641/00063568(2001)051 [0341:LNDATS]2.0.CO;2

Matzke, D., \& Wagenmakers, E.-J. (2009). Psychological interpretation of the ex-Gaussian and shifted Wald parameters: A diffusion model analysis. Psychonomic Bulletin \& Review, 16(5), 798-817. https://doi.org/10.3758/PBR.16.5.798

Moreno, S. O. (2019).facetscales: Facet_grid with different scales per facet. http://github.com/zeehio/facetscales 
Morey, R. D., \& Rouder, J. N. (2011). Bayes factor approaches for testing interval null hypotheses. Psychological Methods, 16(4), 406-419. https://doi.org/10.1037/a0024377

Müller, K., \& Wickham, H. (2018). tibble: Simple Data Frames. https:/ CRAN.Rproject.org/package $=$ tibble

Nieuwenhuis, S., Forstmann, B. U., \& Wagenmakers, E.-J. (2011). Erroneous analyses of interactions in neuroscience: A problem of significance. Nature Neuroscience, 14(9), 11051107. https://doi.org/10.1038/nn.2886

Olive, D. J. (2014). Statistical Theory and Inference. Springer International Publishing.

Pernet, G. R. (2017). Null hypothesis significance testing: A guide to commonly misunderstood concepts and recommendations for good practice. F1000Research, 4, 621. https://doi.org/10.12688/f1000research.6963.5

Pernet, G. R., Wilcox, R. R., \& Rousselet, G. A. (2013). Robust Correlation Analyses: False Positive and Power Validation Using a New Open Source Matlab Toolbox. Frontiers in Psychology, 3. https://doi.org/10.3389/fpsyg.2012.00606

R Core Team. (2021). R: A Language and Environment for Statistical Computing. R Foundation for Statistical Computing. https://www.R-project.org/

Racine, J. S., \& MacKinnon, J. G. (2007a). Simulation-Based Tests that Can Use Any Number of Simulations. Communications in Statistics - Simulation and Computation, 36(2), 357-365. https://doi.org/10.1080/03610910601161256 
Racine, J. S., \& MacKinnon, J. G. (2007b). Inference via kernel smoothing of bootstrap P values. Computational Statistics \& Data Analysis, 51(12), 5949-5957. https://doi.org/10.1016/j.csda.2006.11.013

Roberts, J., \& Fan, X. (2004). Bootstrapping within the multilevel/hierarchical linear modeling framework: A primer for use with SAS and SPLUS. Multiple Linear Regression Viewpoints, 30(1), 23-34. http://www.glmj.org/archives/MLRV_2004_30_1.pdf\#page=24

Rousselet, G. A., Pernet, C. R., \& Wilcox, R. R. (2017). Beyond differences in means: Robust graphical methods to compare two groups in neuroscience. European Fournal of Neuroscience, 46(2), 1738-1748. https://doi.org/10.1111/ejn.13610

Rousselet, G. A., Pernet, C. R., \& Wilcox, R. R. (2019, June 29). The percentile bootstrap: A primer with step-by-step instructions in $R$ [reproducibility package]. https:/ / osf.io/dvuze/

Rousselet, G. A., Pernet, C. R., \& Wilcox, R. R. (2021). The Percentile Bootstrap: A Primer With Step-by-Step Instructions in R. Advances in Methods and Practices in Psychological Science, 4(1), 2515245920911881. https://doi.org/10.1177/2515245920911881

Rousselet, G. A., \& Wilcox, R. R. (2019). Reaction times and other skewed distributions: Problems with the mean and the median [Preprint]. PsyArXiv. https://doi.org/10.31234/osf.io/3y54r

Rubin, D. B. (1981). The Bayesian Bootstrap. The Annals of Statistics, 9(1), 130-134. https://doi.org/10.1214/aos/1176345338 
Ruscio, J., \& Kaczetow, W. (2008). Simulating Multivariate Nonnormal Data Using an Iterative Algorithm. Multivariate Behavioral Research, 43(3), 355-381. https://doi.org/10.1080/00273170802285693

Steel, E. A., Liermann, M., \& Guttorp, P. (2019). Beyond Calculations: A Course in Statistical Thinking. The American Statistician, 73(sup1), 392-401. https://doi.org/10.1080/00031305.2018.1505657

Tintle, N., Chance, B., Cobb, G., Roy, S., Swanson, T., \& VanderStoep, J. (2015). Combating Anti-Statistical Thinking Using Simulation-Based Methods Throughout the Undergraduate Curriculum. The American Statistician, 69(4), 362-370. https://doi.org/10.1080/00031305.2015.1081619

Tukey, J. W., \& McLaughlin, D. H. (1963). Less Vulnerable Confidence and Significance Procedures for Location Based on a Single Sample: Trimming/Winsorization 1. Sankhyā: The Indian Fournal of Statistics, Series A (1961-2002), 25(3), 331-352. JSTOR. https://www.jstor.org/stable/25049278

Venables, W. N., \& Ripley, B. D. (2002). Modern Applied Statistics with S (Fourth). Springer. https://www.stats.ox.ac.uk/pub/MASS4/

Voss, A., Nagler, M., \& Lerche, V. (2013). Diffusion models in experimental psychology: A practical introduction. Experimental Psychology, 60(6), 385-402. https://doi.org/10.1027/1618-3169/a000218 
Wickham, H. (2016). ggplot2: Elegant Graphics for Data Analysis (2nd ed.). Springer International Publishing. https://www.springer.com/gb/book/9783319242750

Wickham, H. (2020). cubelyr: A Data Cube 'dplyr' Backend. https://CRAN.Rproject.org/package $=$ cubelyr

Wilcox, R. R. (2009). Comparing Pearson Correlations: Dealing with Heteroscedasticity and Nonnormality. Communications in Statistics - Simulation and Computation, 38(10), 2220-2234. https://doi.org/10.1080/03610910903289151

Wilcox, R. R. (2016). Comparing dependent robust correlations. The British Fournal of Mathematical and Statistical Psychology, 69(3), 215-224. https://doi.org/10.1111/bmsp.12069

Wilcox, R. R. (2017). Introduction to Robust Estimation and Hypothesis Testing (4th edition). Academic Press.

Wilcox, R. R., \& Keselman, H. J. (2003). Modern robust data analysis methods: Measures of central tendency. Psychological Methods, 8(3), 254-274. https://doi.org/10.1037/1082989X.8.3.254

Wilcox, R. R., \& Rousselet, G. A. (2018). A Guide to Robust Statistical Methods in Neuroscience. Current Protocols in Neuroscience, 82(1), 8.42.1-8.42.30. https://doi.org/10.1002/cpns.41

Wilke, C. O. (2017). cowplot: Streamlined Plot Theme and Plot Annotations for 'ggplot2'. https:/ /CRAN.R-project.org/package= cowplot 
Wu, C. F. J. (1986). Jackknife, Bootstrap and Other Resampling Methods in Regression Analysis. The Annals of Statistics, 14(4), 1261-1295. https://doi.org/10.1214/aos/1176350142

Xie, Y. (2018). knitr: A General-Purpose Package for Dynamic Report Generation in R. https://yihui.name/knitr/

Yan, Y., \& Genton, M. G. (2019). The Tukey g-and-h distribution. Significance, 16(3), 12-13. https://doi.org/10.1111/j.1740-9713.2019.01273.x 